\title{
Acknowledgement to Reviewers of International Journal of Molecular Sciences in 2016
}

\author{
International Journal of Molecular Sciences Editorial Office \\ MDPI AG, St. Alban-Anlage 66, 4052 Basel, Switzerland; ijms@mdpi.com \\ Published: 11 January 2017
}

The editors of International Journal of Molecular Sciences would like to express their sincere gratitude to the following reviewers for assessing manuscripts in 2016.

We greatly appreciate the contribution of expert reviewers, which is crucial to the journal's editorial process. We aim to recognize reviewer contributions through several mechanisms, of which the annual publication of reviewer names is one. Reviewers receive a voucher entitling them to a discount on their next MDPI publication and can download a certificate of recognition directly from our submission system. Additionally, reviewers can sign up to the service Publons (https://publons.com) to receive recognition. Of course, in these intiatives we are careful not to compromise reviewer confidentiality. Many reviewers see their work as a voluntary and often unseen part of their role as researchers. We are grateful to the time reviewers donate to our journals and the contribution they make.

If you are interested in becoming a reviewer for International Journal of Molecular Sciences, see the link at the bottom of the webpage http://www.mdpi.com/reviewers.

The following reviewed for International Journal of Molecular Sciences in 2016:

\author{
Abbott, Karen L. \\ Abdelmohsen, Kotb \\ Abdel-Wahab, Omar \\ Abdulah, Rizky \\ Abe, Sumiko \\ Abel, Peter \\ Abenavoli, Ludovico \\ Abizaid, Alfonso \\ Ablonczy, Zsolt \\ Abraham, Wolf-Rainer \\ Abranches, Rita \\ Abriata, Luciano A. \\ Aburima, Ahmed \\ Achinger-Kawecka, Joanna \\ Achsel, Tilmann \\ Adachi, Hiroaki \\ Adamakis, I.-D.S. \\ Adamcakova-Dodd, Andrea \\ Adamo, Vincenzo \\ Adams, James \\ Adegoke, O.A.J. \\ Adoro, Stanley \\ Adunyah, Samuel E. \\ Afantitis, Antreas \\ Afrin, Sadia
}

Agarwal, Neeraj
Agger, Jane W.
Agodi, Antonella
Agon, Vanessa
Agrawal, Alok
Agrawal, Sandeep K.
Agrimson, Kellie
Agulló-Ortuño, M. Teresa
Agut, Montserrat
Ahi, Yadvinder
Ahmad, Zeeshan
Ahmed, Ammar M.
Ahmed, Nisar
Ahrens, Christian
Aikawa, Takuya
Aires, Alfredo
Airoldi, Cristina
Akahane, Manabu
Akamatsu, Miki
AKASHI, Makoto
Akdemir, Deniz
Akitsu, Takashiro
Akopian, Armen N.
Alam, Antoine
Alander, Jarmo

\author{
Alary, Fabienne \\ Alavi, Saman \\ Albahrani, Ali A. \\ Albano, Emanuele \\ Albericio, Fernando \\ Alberti, Saverio \\ Albinsson, Sebastian \\ Al-Dujaili, Emad \\ Alenghat, Francis J. \\ Alessandro, Riccardo \\ Alexandre-Gouabau, M.-C. \\ Alexiou, Christoph \\ Alfaidy, N. \\ Alfano, Daniela \\ Alfano, Massimo \\ Alfonzo, Antonio \\ Alfredsson, Jenny \\ Al-Horani, Rami \\ Aliev, Fazil \\ Alkilani, Ahlam Zaid \\ Allegaert, Karel \\ Almajano, María Pilar \\ Almasio, Piero Luigi \\ Almstrup, Kristian \\ Aloisi, Anna Maria
}


Alonso, Conchita

Alonso-Alconada, Daniel

Alpini, Gianfranco

Alsaweed, Mohammed

Alsteens, David

Alt, David P.

Alteri, Christopher J.

Altintas, Zeynep

Aluwini, Shafak

Alvarez, Carlos

Alvarez-Suarez, José Miguel

Álvaro, Tomás

Alves, Celso

Alves, Sandra

Alwahsh, Salamah M.

Alyokhin, Andrei

Alzaid, F.

Amant, Carole

Amar, Laurence

Amaral, Alexandra

Amasheh, Salah

Ambe, Kimiharu

Ambe, Peter C.

Ambigapathy, Ganesh

Amicarelli, Fernanda

Amital, Howard

Ammendola, Rosario

Amorati, Riccardo

Amorós, Asunción

Anczukow-Camarda, Olga

Andersen, Bogi

Andersen, Morten

Andersen, Thomas Levin

Anderson, Anne J.

Anderson, Travis M.

Andersson, Håkan S

Andersson, Leif C.

Andersson, Mats X.

Andrade, Fernando

Andrade, Paula

André, Vânia

Andrea, Onofri

Andreadou, Ioanna

André-Fontaine, Genevieve

Andreoni, Francesca

Andreozzi, Giuseppe Maria

Andres, Allen M.

Andrukhov, Oleh

Anes, Elsa

Angeletti, Mauro

Angelico, Francesco

Angelini, Claudia
Angelone, Tommaso

Angelucci, Adriano

Angulo, Javier

Aniello, Francesco

Anifandis, George

Annunziata, Pasquale

Anraku, Makoto

Ansorena, Diana

Ansquer, Jean Claude

Antaris, Alexander Lee

Anthony, Karen

Antognelli, Cinzia

Antoni, Delphine

Antoniadi, Ioanna

Antoniak, Silvio

Aon, Miguel A.

Aparicio, Frederic

Aperia, Anita

Appetecchi, G.B.

Aprea, Eugenio

Apte, Swapna

Apte, Udayan

Arai, Ryoichi

Araniti, Fabrizio

Arasaradnam, Ramesh P.

Arbefeville, Sophie

Arbona, Vicent

Archer, Trevor

Archibald, Steve

Arcidiacono, Biagio

Ardley, Julie

Arduini, Alessandro

Arens, Paul

Arese, Marzia

Ariani, Andrea

Ariga, Katsuhiko

Arnhold, Juergen

Aroca, Ricardo

Arpawong, Thalida E.

Arrick, Denise

Arrighi, Silvana

Arroyo, Cristina

Arthur, Michel

Artlip, Timothy

Arul, Joseph

Asa, Sylvia L.

Asahina, Kinji

Asakura, Tetsuo

Asano, Ryutaro

Asard, Han

Ascher, David B.

Asensi, V.
Ashiuchi, Makoto

Asimakopoulos, A.G.

Assfeld, Xavier

Astolfi, Stefania

Atanasov, Atanas G.

Atanassova, Rossitza

Atangana, Alain R.

Ates-Alagoz, Zeynep

Athyros, Vasilios

Atkinson, Sarah

Atluri, Venkata S.R.

Atrian, Silvia

Atsawasuwan, Phimon

Attanasio, Chiara

Attardo, Geoffrey M.

Audet, Céline

Auguet, Teresa

Aury-Landas, Juliette

Austin, Brian

Austin, Caroline

Autelli, Riccardo

Avdic, Selmir

Averbeck, Dietrich

Avola, R.

Avrova, Natalia F.

Aylon, Yael

Ayoub, Nadia A.

Ayyavoo, Velpandi

Azarbal, Amir F.

Azarnia Tehran, Domenico

Azevedo, Helena

Azmi, Asfar Sohail

Azoitei, Ninel

Azuma, Masaaki

Baba, Hideo

Babajko, Sylvie

Babu, Anish

Babu, Dinesh

Baburamani, Ana

Bache, Søren

Backus, Deborah

Bae, Yoe-Sik

Baechlein, Chrsitine

Baeg, Gyeong Hun

Baer, Patrick C.

Bagby, Michael

Bagni, Claudia

Bai, Li-Yuan

Bai, Wei

Bailard, Neil S.

Bairaktari, Eleni T.

Baisantry, Arpita 
Bajpai, Richa

Baker, Simon C.

Baksh, Shairaz

Balandraud, Nathalie

Balatti, Pedro

Balcells, Mercedes

Baldacci-Cresp, Fabien

Baldermann, Susanne

Baldoni, Elena

Balduini, Walter

Baldwin, Scott

Balestrini, Raffaella Maria

Balietti, Marta

Ball, Jonathan

Ballerini, Clara

Ballestri, Stefano

Ballottari, Matteo

Baltazar, Fátima

Baluska, Frantisek

Bamford, Connor

Banack, Sandra

Banchero, Mauro

Banerjee, Areen

Banerjee, Hirendra Nath

Banerjee, Jheelam

Banerjee, Kalpita

Banerjee, Pratik

Baniahmad, Aria

Banilas, Georgios

Banskota, Arjun H.

Banti, Christina N.

Bao, Guanhui

Bapputty, Reena

Baptiste-Roberts, Kesha

Barabutis, Nektarios

Baratta, Francesco

Barba, Francisco

Barba, Maddalena

Barbagallo, Davide

Barbas, Coral

Barbayianni, Efrosini

Barberi, Tiziano

Barbier, Olivier

Barbieri, Federica

Barbosa-Leiker, Celestina

Bariana, Harbans

Barnea, Eytan R.

Barone, Flavia

Barradas, Didier

Barreira, Luísa

Barros, Pedro

Bar-Shavit, Rachel
Barta, Csengele

Bartfeld, Sina

Bartha, István

Bartoli, Francesco

Barzilay, Joshua I.

Basak, Debasish

Basaraba, Randall J.

Basavarajappa, Balapal

Bashan, Yoav

Basini, Giuseppina

Bassil, Nahla V.

Basso, Kari

Bastola, Dhundy R.

Basu, Abhijit

Basu, Sutapa

Batail, Patrick

Bates, Darcy

Battaglia, Agatino

Battistelli, Michela

Bauer, Martin

Baum, Christel

Baum, Jamie

Baum, Michel

Baumann, Arnd

Baumann, Heinz

Baumgärtner, Wolfgang

Bavilacqua, Antonio

Bayliss, Sion

Bazhin, Alexandr

Bazylińska, Urszula

Becana, Manuel

Becerra, S. Patricia

Bechmann, Ingo

Beck, Dominik

Beck, William

Becker, Christoph

Beckmann, Manfred

Becuwe, Philippe

Bede, Jacqueline C.

Bedia, Carmen

Bedini, Emiliano

Bednarski, Patrick

Beggs, Simon

Behera, Smrutisanjita

Beilby, Mary

Beitz, Eric

Bela-Ong, Dennis

Beldi, Guido

Belkacemi, Louiza

Bell, Stephen

Bella, Federico

Bellés, José María
Bellezza, Ilaria

Bello, Salvador

Bello, Xanel Vecino

Bellomo, Elisa A.

Belmadani, Souad

Beloqui, Ana

Belstrøm, Daniel

Belyaeva, Natalia

Bendahhou, Saïd

Benedetti, Michele

Benediktsdóttir, Berglind Eva

Beney, Laurent

Bénichou, Serge

Benito, Juan M.

Benjak, Andrej

Benoist, Hervé

Benoit, Danielle S.W.

Ben-Shimol, Shalom

Benson, Al B.

Benzeroual, Kenza E.

Berchtold, Martin

Bercion, Sylvie

Beretta, Giovanni

Bergen, Werner

Berger, Kenneth I.

Berkowitz, Bruce A.

Berland, Sophie

Berlanga, Ángel Mérida

Berlin, K. Darrell

Bermudez, Marcel

Bernardi, Rafael C.

Bernards, Matt

Bernasconi, Pia

Bernt, Kathrin

Bernt, Matthias

Berron, Brad

Bertino, Massimo F.

Bertolini, Marta

Bertoni, Giuseppe

Bertram, Ralph

Besada, Cristina

Besalú, Emili

Besson, Thierry

Bethea, Cynthia L.

Betoret, Ester

Bettaieb, Ali

Bettington, Mark

Bevilacqua, Antonio

Bhagwat, Nikhil

Bhat, Shridhar

Bhattacharyya, Gargi

Bhatwadekar, Ashay D. 
Bhonde, Ramesh

Ramchandra

Bhutoria, Savita

Bi, Enguang

Biasini, Emiliano

Bickle, Marc

Bid, Hemant K.

Biddle, Adrian

Biegstraaten, Marieke

Bienert, Gerd P.

Biernasiuk, Anna

Biersack, Bernhard

Bilbao, Jose Ramon

Bill, Roslyn M.

Birch, John

Birrell, Mark

Bishop, Karen

Bisht, Vikram

Bita, Craita E

Bitter, Istvan

Bix, Gregory

Bizzarri, Mariano

Björkbacka, Harry

Björnsson, Einar

Blackburn, Daniel G.

Blagden, Sarah

Blair, Paul

Blais, Anne

Blaker, Jonny

Blamires, Sean J.

Blanco, José Manuel Lopez

Blandino, Giovanni

Blando, Federica

Blank, Michael

Błaszczyk, Lidia

Blattner, C.

Bleackley, Mark R.

Blesso, Christopher

Bloemers, Jos

Board, Mary

Bobe, Régis

Boccuto, Luigi

Bock, C.-Thomas

Bode, Robert F.

Bodenstine, Thomas

Bodnar, Richard

Boeckh-Behrens, $\mathrm{T}$.

Boelsterli, Urs A.

Boer, Judith M.

Bogan, Jonathan S.

Bögemann, Martin

Bogni, Alessia
Bogomolnaya, Lydia M.

Boguszewicz, Ł.

Bøgwald, Jarl

Böhm, Volker

Bohutskyi, Pavlo

Bohutskyi, Pavlo

Boisvert, François-Michel

Boka, Vasiliki-Ioanna

Bold, Richard J.

Bolle, Cordelia

Bollini, Sveva

Bolmont, Tristan

Boltje, Thomas J.

Bolukbasi, Fatih

Bombarda, Isabelle

Bombelli, Paolo

Bomben, Riccardo

Bomprezzi, Roberto

Bonacina, Luigi

Bonaventure, Pascal

Bonfield, Tracey L.

Bonikowski, Radosław

Bonventre, Joseph V.

Boonkaew, Benjawan

Booth, Brian

Bora-Singhal, Namrata

Borbély, Yves

Bordignon, Vilceu

Borghini, Andrea

Borkovich, Katherine A.

Bormann, Jörg

Borole, Abhijeet P.

Borovsky, Dov

Borràs, Francesc E.

Borrás-Hidalgo, Orlando

Borroni, Riccardo

Borroto-Escuela, Dasiel

Bosch, Christine

Bosco, Domenico

Bose, Debojit

Bose, Utpal

Boselli, Emanuele

Boskou, Dimitrios

Botella, Jimmy

Both, Sanne

Bottari, F.

Bottino, Marco C.

Böttner, Martina

Bouaïcha, Noureddine

Boulter, Luke

Bouquillon, Sandrine

Bourdon, Jean-Christophe
Bourgeois, Dominique

Boutinguiza, Mohamed

Bove, Kevin E.

Bowater, Richard

Bowen, Richard A.

Bowling, Heather

Box, Neil

Boyd, Mark

Boyom, Fabrice Fekam

Braathen, Lasse

Bradley, Walter G.

Braeuning, Albert

Bragado, M.J.

Brait, Mariana

Bramanti, Vincenzo

Brambilla, Eugenio

Brambilla, Vittoria

Branchini, Alessio

Brand, Luise

Brás, Natércia

Brastianos, Priscilla K.

Braunstein, Evan M.

Bravo López, Susana B.

Bravo, Jeronimo

Breckenridge, Ross

Brecker, Lothar

Breckpot, Karine

Brehme, Marc

Breitenfeld Granadeiro, Luiza

Brender, Jeffrey R.

Brennan, Frank

Bresson, Justine

Brewer, Gary

Brewin, Nick

Bricknell, Ian

Bridgewater, Darren

Briedé, Jacco

Brier, Michael E.

Briles, David L.

Brinchmann, Monica F.

Brites, Carla

Briza, Peter

Broadbent, Andrew

Brochier, Camille

Broderick, Lori

Broderick, Tom L.

Brona, Murphy

Bronisz, Agnieszka

Brooks, Amanda E.

Brooks, Anna

Brossaud, Julie

Brough, David 
Brough, Louise

Broussard, Josiane L.

Brown, Geoffrey

Brown, James

Brown, Lewis

Brown, Neil Andrew

Bruce, Erica D.

Bruining, Nico

Brummelte, Susanne

Brun, Nicolas

Brundo, Maria Violetta

Brunelleschi, Sandra

Brunetti, Cecilia

Brunker, Kirstyn

Brunner, Gerd

Brusselaers, Nele

Brustmann, Hermann

Bryson-Richardson, Robert

$\mathrm{Bu}$, Xianhe

Bubici, Giovanni

Buccheri, Marina

Buckley, M.

Bucolo, Claudio

Budak, Hikmet

Buechler, Christa

Buenzli, Pascal R.

Bugg, Timothy D.H.

Bühling, Frank

Buján, Noemí

Bullock, Anthony

Bullon, Pedro

Bunimovich, Yuri L.

Burghardt, Robert

Burke, Mark

Burse, Antje

Bus, James $S$.

Busardo, Francesco

Buscarinu, Maria Chiara

Busch, Hauke

Bushley, Kathryn

Bustamante, Alejandro

Butlin, Mark

Caballero, Julio

Cabanillas, Beatriz

Cabarcos, Pamela

Cabral, Horacio

Cabrera-Fuentes, Hector

Caceres, Alejandro

Caffarel, María Muñoz

Caffarelli, Carlo

Cahalon, Liora

Cahill, Abigail E.
Cahoon, Aubrey Bruce

Cai, Lu

Cairncross, Carolyn

Cairo, Gaetano

Cairrão, Elisa

Calado, Ângelo

Calaf, Gloria M.

Calcagno, A.

Calcutt, Michael

Calì, Tito

Calokerinos, Antony

Calorini, Lido

Calvisi, Diego F.

Calzavara-Pinton, P.

Calzetta, Luigino

Camarda, Kyle

Cambier, Sébastien

Cameli, Norma

Campanale, Joseph P.

Campanella, Michelangelo

Campanero-Rhodes, M.A.

Campbell, Christopher

Campbell, Lee

Campbell, Michael

Campello, Silvia

Campione, Marina

Camporeale, Annalisa

Campos Rosa, Joaquín

Cañada, F. Javier

Canini, Antonella

Cañizares, Carmen

Cannavo, Elda

Cao, Ling

Caparrotta, Laura

Capel, Frédéric

Capitanio, Nazzareno

Capodaglio, Andrea

Cappelletti, Vera

Capperucci, Antonella

Caprari, Patrizia

Caramella, Carla

Carapelli, Antonio

Carattino, M.D.

Carbajo-Pescador, Sara

Carcillo, Joseph

Cardarelli, Francesco

Cardin, Christine

Carlinfante, Gabriele

Carlomagno, Chiara

Carnevale, Gianluca

Carol, Pierre

Caroli, Manuela
Carpenter, Margaret A.

Carr, Carolyn

Carr, John P.

Carranca, Corina

Carraro, Mauro

Carrasco, Pedro

Carregal-Romero, Susana

Carreras, Isabel

Carrì, Maria Teresa

Carroll, Ronan

Carrubba, Alessandra

Carter, Wayne G.

Caruso-Nicoletti, Manuela

Carvajal, Micaela

Carvalho, Ana

Carvalho, Luísa

Casas, Ana M.

Casey, Alan

Cash, Phillip

Casini, Giovanni

Caskey, Marina

Casquero, Pedro A.

Castaño, Oscar

Casteel, Darren E.

Castell, Alina

Castellano, Orlando

Castellanos-Garzón, José A.

Castiglioni, Bianca

Castillo, Pablo

Castñeiras, Alfonso

Caswell, Clayton C.

Cataldi, T.R.I.

Catanzano, Ovidio

Catto-Smith, Anthony

Catts, Vibeke

Cauffiez, Christelle

Cauli, Omar

Cavalla, P.

Cavalli, Roberta

Caviglia, Gian Paolo

Ceasar, S.A.

Ceccarelli, Sara

Cehofski, Lasse

Celli, Anna

Cellinese, Nico

Cerchia, Laura

Ceriotti, Aldo

Ceroni, Dimitri

Ceroni, Fabiola

Cerretani, Lorenzo

Cervantes, Jorge L.

Cesare Marincola, Flaminia 
Cesselli, Daniela

Chaban, Christina

Chae, Han-Jung

Chai, Han-Ha

Chaidos, Aristeidis

Chakrabarti, Ratna

Chakraborty, Anirban

Challacombe, Jean F.

Challet, Etienne

Chamcheu, Jean Christopher

Chami, Belal

Chan, Aden K.Y.

Chan, Danny

Chan, King Ming

Chan, Kun-Ming

Chan, Thomas

Chand, Sourabh

Chandaka, Giri Kumar

Chang, Cheng

Chang, Chia M.

Chang, Chien-Chung

Chang, Chih Long

Chang, Chin-Chyuan

Chang, Ching-Jin

Chang, Chin-Sung

Chang, Chun-Che

Chang, Fang-Rong

Chang, Hen-Hong

Chang, Hsin-I

Chang, Ing-Feng

Chang, Jin Ho

Chang, Jungshan

Chang, Ko-Tung

Chang, Lin-Li

Chang, Long-Sen

Chang, Shun-Fu

Chang, Te-Sheng

Chang, Wei-Chiao

Chang, Wen-Chi

Chang, Wen-Wei

Chao, Thomas

Chapel, Alain

Charchar, Fadi

Charlton, Clivel

Chassaing, Benoit

Chater, Caspar

Chatterjee, Jhinuk

Chatzizisis, Yiannis S.

Chaudhry, Parvesh

Chauhan, Arun

Chaves-Pozo, Elena

Chelland Campbell, Sara
Chen, Banglin

Chen, Chao-Jung

Chen, Cheng-Lung

Chen, Chi

Chen, Chih-Cheng

Chen, Chiing-Chang

Chen, Chongyi

Chen, Chung-Yi

Chen, Chun-Jung

Chen, Huaiqiong

Chen, Huaping

Chen, Jiann-Chu

Chen, Jiao

Chen, Jih-Jung

Chen, Kevin

Chen, $\mathrm{Li}$

Chen, Miaw-Ling

Chen, Po-Chia

Chen, Qi

Chen, Qi-Yin

Chen, Tianbao

Chen, Tzong-Yueh

Chen, Xing

Chen, Y.-M.

Chen, Yei-Tsung

Chen, Yen-Hao

Chen, Yi-Hung

Chen, $\mathrm{Yu}$

Chen, Yu-Ching

Chen, Yuhang

Chen, Yung-Chang

Chen, Yun-Wen

Chen, Zhe-Sheng

Chen, Zhihang

Chen, Zhong

Cheng, Fong-Yu

Cheng, Heung-Chin

Cheng, Jing-Jy

Cheng, Juei-Tang

Cheng, Kuang-Hung

Cheng, S.S.

Cheng, Ting-Yuan David

Cheng, Yong

Cheon, Yong-Pil

Cheong, Yong Hwa

Cherbuy, Claire

Cheriyath, Venugopalan

Chermette, Henry

Chern, Ming-Kai

Chevali, Venkata

Chevallier, Nathalie

Chew, Li-Jin
Cheynier, Véronique

Chhatbar, Pratik

Chiang, Hsiu-Mei

Chiang, Meng-Tsan

Chiang, Tai-An

Chiang, Yu-Chung

Chiba, Yoshihiko

Chien, Hung-Yu

Chiesa, Marco

Chiessi, Ester

Childs, Gwendolyn Vaughn

Chilton, Robert

Chim, Stephen

Chimenti, Sergio

Chin, Michael

Ching, Wei-Mei

Chinnam, Parameswara Rao

Chiou, Chun-Tang

Chirumbolo, Salvatore

Chisholm, John D.

Chitarra, Walter

Chiu, Ing-Ming

Chiu, Ka Fung Peter

Chiurazzi, Maurizo

Chiurchiù, Valerio

Chiva-Blanch, Gemma

Cho, Hyun-Jeong

Cho, Ssang-Goo

Chobot, Vladimir

Choi, Eun-Kyoung

Choi, Eun-Mi

Choi, Hyung-Kyoon

Choi, Jang Hyun

Choi, Jor-Shan

Choi, Woonyoung

Choi, Yoo Seong

Choi, Yung Hyun

Chomilier, Jacques

Chong, Parkson Lee-Gau

Chopp, Michael

Chorianopoulos, Nikos G.

Chou, Angela

Chou, Kuo-Chen

Chou, Wing-Ming

Choudhury, Malay

Chougule, Mahavir

Chowdhury, Kamal

Chren, Mary Margaret

Christiaens, Olivier

Christofidou-Solomidou, M.

Chrzanowski, Łukasz

$\mathrm{Chu}$, Chun-Hung 
Chu, Dafeng

Chu, Shidong

Chua, Melvin

Chuang, Chin-Kai

Chuang, Lea-Yea

Chuang, Yung-Jen

Chudasama, Vijay

Chueh, Anderly

Chun, Byung-Soo

Chun, Kyung-Soo

Chung, Chuhan

Chung, Eun Joo

Chung, Yong-An

Chyau, Charng-Cherng

Ciaccio, Marcello

Ciaudo, Constance

Ciccacci, Cinzia

Ciccozzi, Massimo

Cicero, Arrigo

Cichero, Elena

Cignetti, Alessandro

Cilia, Michelle

Cimino, Filiberto

Cindrova-Davies, Tereza

Ciogli, Alessia

Cipolla, David

Cirillo, Giuseppe

Cisowsky, Jaroslaw

Citro, Valentina

Citterio, Davide

Ciulu, Marco

Clark, Lindsay V.

Claus, Harald

Clausen, Rasmus Prætorius

Clawson, Gary A.

Clem, Brian F.

Cleveland, Beth M.

Clevenger, Charles V.

Clowry, Gavin J.

Coan, P.M.

Coates, Christopher J.

Coccheri, Sergio

Cocetta, Giacomo

Cochran, Blake

Coco, Silverio

Codacci-Pisanelli, Giovanni

Coelho, José A.

Cognasse, Fabrice

Cohen, Dan

Cohrs, Randall

Cojocaru, Vlad

Cole, John W.
Coleman, William B.

Collavin, Licio

Collawn, Sherry S.

Collin, Matthew

Collin, Rob W.J.

Colloc'h, Nathalie

Collongues, Nicolas

Colombo, Gualtiero I.

Colson, Natalie

Colvin, Robert

Comai, Stefano

Conant, Katherine

Conboy, John

Conigliaro, Alice

Conlan, Sean

Connelly, Kim A.

Conner, Matthew

Contassot, Emmanuel

Conte, Enrico

Conte, Sasi

Cook, Atlanta G.

Cook-Mills, Joan M.

Cooney, Austin

Cooney, Ralph

Cooper, Arthur

Cooper, Timothy K.

Cooper, William T.

Copple, Ian

Corbett, Harriet J.

Corbetta, Sabrina

Corcoran, Brendan M.

Cordat, Emmanuelle

Cordero, Paul

Cornelissen, Bart

Corniola, Rikki

Correia, Sofia

Correia-de-Sá, Paulo

Correia-Neves, Margarida

Corrigan, Frances

Corsetto, Paola A.

Cortese, Francesca

Cortese, Katia

Ćosić, Jasenka

Cosset, François-Loïc

Costa, Catalina Martinez

Costa, Fabrizio

Costa, José Luis

Costa, Paulo J.

Costa, Rui

Costagliola, Ciro

Costantino, Luca

Côté, Jean-François
Coto, Eliecer

Coulter, Jonathan A.

Coupland, Lindsay J.

Courtney, Brian K.

Courty, Pierre-Emmanuel

Courville, Elizabeth L.

Cousin, Xavier

Couttet, Philippe

Coutts, Robert

Coyle, Beth

Craig, Andrew W.B.

Craig, Timothy P.

Crava, Cristina M.

Cray, James J.

Crespi, Martin

Crisafulli, Concetta

Cristiani, Pierangela

Croce, Anna Cleta

Croset, Martine

Cross, Carroll E.

Crotti, Tania

Crowe, David R.

Cuesta-Seijo, Jose A.

Cui, Haitao

Cui, Juan

Culot, Maxime

Culotta, Valeria C.

Cummings, Brian

Cunningham, Patrick

Curtin, Chris

Curtis, Wayne

Czosnek, Henryk

Czosnyka, Marek

D'Anna, Francesca

D'Souza-Schorey, Crislyn

Da Cruz-Höfling, Maria Alice

Da Silva, A. Moreira

D'Abusco, Anna Scotto

Dacher, Matthieu

Dacheux, Jean-Louis

Daftarian, Pirouz

Dag, Arnon

Dagnino, Lina

Dague, Etienne

Dahaba, Ashraf A.

Dai, Shaojun

Dai, Shu-Mei

Daidone, Maria Grazia

Dailey, Harry

Dailianis, Stefanos

Dall'Acqua, Stefano

Dall'Olio, Fabio 
Damaraju, Sambasivarao

Damia, Giovanna

Damico, Michele

Da'na, Enshirah

Danan, Gaby

Dandekar, Thomas

Dane, Fenny

D’Angelo, Livia

D'Angiolella, Vincenzo

Danielson, Kirstie K.

Danielson, Neil

Danila, Daniel C.

Danilenko, Michael

Darby, Ian A.

Dare, Andrew

Dare, Ryan

D'Argenio, Giuseppe

D'Argenio, Valeria

Dark, Michael J.

Darland, Tristan

Darling, Seth

Darvesh, Sultan

Darvin, Maxim

Das, Anindita

Das, Arup

Dasgupta, Santanu

Dashti, Hassan

Davenport, Anthony

Davenport, Mark

Daverey, Amita

Davey, Rachel A.

Davidson, Nicholas O.

Davies, Keith

Davies, Kelvin J.A.

Davies, Kevin

Davies, Leo

Davies, Michaell

Davis, Gregory D.

Davis, Mindy

Davolos, Domenico

De Aberasturi, D.J.

De Andrés Cara, Damián F.

De Berardis, Domenico

De Bernonville, T.D.

De Biase, Dario

De Bortoli, Michele

De Cáceres González, F.F.N.

De Feo, Vincenzo

De Francia, Silvia

De Geest, Bart

De Gelder, Leen

De Giorgi, Ugo
De Guzman Strong, Cristina

De Jong, Jill L.O.

De Jonghe, Kris

De Koning, Dirk

De Kort, Laetitia M.O.

De Kretser, David Morritz

De La Cruz, José Pedro

De La Herrán, Roberto

De La Hoz, Antonio

De La Luz Cádiz Gurrea, M.

De La Monte, Suzanne

De La Torre, Xavier

De Lago, Eva

De Lange, Pieter

De Mattei, Monica

De Morais, R.M.S.C.

De Noronha, Carlos M.C.

De Paolis, Angelo

De Rivero Vaccari, Juan Pablo

De Ron, Antonio

De Rosa, Maria Cristina

De Rosa, Viviana

De Visser, S.P.

De Vos, John

De Vos, Ric

De Vries, Carlie

De, Kuntal

Dearden, John C.

Debode, Frédéric

Debray, François-Guillaume

Decker, Michael

Deeg, Cornelia

Deevska, Gergana

Degano, Ilaria

Degens, Hans

Deive Herva, Francisco J.

Dekaban, Gregory A.

Dekker, Frank

Dekker, Marloes

Del Álamo, M.M.R.

Del Vescovo, Valerio

Delagoutte, Emmanuelle

Delerue, Fabien

Delgado, María Jesús Santos

Delhanty, Patric J.D.

Delisle, Hélène

Dell'Era, Patrizia

Delporte, Christine

DelRosso, Mario

Demitrack, Elise

Demorrow, Sharon

Deng, Qing
Deng, Xufang

Deng, Yong

Deng, Yongming

Denizot, B.

Dennenmoser, Stefan

Dentino, Andrew R.

Dermauw, Wannes

DesBordes, Charles

Deshayes, Sébastien

Desmaële, Didier

Desmet, Eline

Dessy, Chantal

Devesa, V.

Devy, Jérôme

Dhaenens, Clarisse

Dhanasekaran, Saravana M.

Dhar, Animesh

Dhaubhadel, Sangeeta

Dhondt-Cordelier, Sandrine

Dhoot, Gurtej K.

Di Benedetto, Barbara

Di Fazio, Pietro

Di Fiore, Maria Maddalena

Di Giannantonio, $\mathrm{M}$.

Di Giovanni, Simone

Di Liegro, Italia

Di Luccia, Aldo

Di Maio, Ernesto

Di Paolo, Antonello

Di Pietro, Cinzia

Di Primio, R.

Di Sansebastiano, Gian Pietro

Di Stefano, Antonio

Diapari, Marwan

Dias, Juliana

Diaz, Isabel

Diaz, Mayri A.

Díaz-Sala, Carmen

Diccianni, Mitchell B.

Dickert, Franz

Dickstein, Rebecca

Didonna, Alessandro

Diegelmann, Robert

Dieplinger, Benjamin

Dieterlen, Maja-Theresa

Dievart, Anne

Dikalov, Sergey

Dimova, Elitsa

Ding, Baoqing

Dingwall, Andrew K.

Dinh, Dzung H.

Dinwiddie, Darrell L. 
Dionisi, Davide

Dionisio, Giuseppe

Diot, Christian

Dioum, Elhadji M.

D'Ischia, Marco

Divi, Rao L.

Dixon, David A.

Djouadi, Fatima

Doan, Ngoc Duc

Dobens, Leonard L.

Doddapaneni, Ravi

Doh, Kyung-Oh

Dolferus, Rudy

Dolga, Amalia

Dolga, Amalia M.

Doll, Kenneth

Dolo, Vincenza

Dombrowski, Nina

Domingos, Pedro M.

Donato, Rosario

Dondelinger, Yves

Dong, Lanfeng

Donly, B. Cameron

Donnini, Sandra

Donzelli, Elisabetta

Donzelli, Sabrina

Doonan, James

Doran, Michael

Dorfman, Damian

Dormond, Olivier

Dorner, A

Dossena, Marta

Douros, Antonios

Doyle, Hester

Doyle, Siamsa

Dragsted, Lars

Drakoulis, Nikolaos

Draper, Isabel

Dreij, Kristian

Drickamer, Kurt

Driscoll, James J.

Drosten, Matthias

Dryga, Anatoly

Du, Dongliang

Du, K.-L.

Duangthip, Duangporn

Duarte, Julio D.

Dubey, Ashish

Dubey, Ramin

Dubielecka, Patrycja M.

DuBois, Debra C.

Dubrova, Yuri
Dudás, József

Dudziak, Diana

Duennwald, Martin L.

Duerksen-Hughes, Penelope

Dufau, Maria L.

Duhamel, Jean

Duman-Scheel, Molly

Dumez, Sylvain

Dumon, Stephanie

Dunietz, Barry D.

Dunne, Eileen M.

Duong, Hai M.

Dupureur, Cynthia M.

Durán, I.

Durazzo, Alessandra

Durbec, Pascale

Duressa, Dechassa

Duszenko, Michael

Dutcher, James D.

Dwivedi, Chandradhar

Dwyer-Nield, Lori D.

Ealy, Alan

Eamens, Andy

Easmon, Johnny

Ebrahim, Weaam

Echevarría, Miriam

Ecke, Thorsten

Eckel, Jürgen

Economou, Christina N.

Edelman, Arthur M.

Eder, Iris E.

Eder, Matthias

Edgren, Tomas

Edin, Nina Jeppesen

Eduardo-Figueira, Maria

Edwards, Jeffrey G.

Ee, Pui-Lai Rachel

Eferl, Robert

Efird, Jimmy

Egawa, Gyohei

Egea, Marcos

Eggeling, Lothar

Eggert, Jullie

Ehlting, Christian

Ehret, Georg B.

Ehrich, Marion

Ehrnhoefer, Dagmar

Eid, Nabil

Eilertsen, Karl-Erik

Eisenreich, Andreas

Eitsuka, Takahiro

Ekinci, Elif I.
Ekuni, Daisuke

El Benna, Jamel

El Desoky, Ehab S.

Elahi, Shokrollah

Elbayoumi, Tamer

Eldeeb, Mohamed

Elez-Martinez, Pedro

Elhanafi, Ahmad Omar

Eliseev, Roman A.

El-kereamy, Ashraf

Elkouby, Yaniv M.

Eller, Fred

Ellies, L.G.

Ellinger, Jörg

Elliott, Paul R.

Ellis, John

Ellsworth, Darrell L.

El-Matbouli, Mansour

El-Mowafy, Omar

Elsea, Sarah H.

El-Seedi, Hesham R.

El-Soda, Mohamed

El-Sohemy, Ahmed

Emile, Jean-François

Emmert, Steffen

En Henegouwen, P.V.B.

Engberg, Göran

Engel, Andrew

Engelberg, David

Engelberth, Jurgen

Engelund, Morten B.

English, Niall

Engström, Maria

Enguita, Francisco

Eppler, Elisabeth

Epstein, Alan

Equils, Ozlem

Erdbruegger, Uta

Eri, Raj

Eriksson, Oskar

Eritja, Ramon

Ersvær, Elisabeth

Escames, Germaine

Esguerra, Jonathan Lou S.

Espen, Luca

Esperança, José M.S.S.

Espinosa-Diez, Cristina

Esposito, Ciro

Esposito, Debora

Esposto, Sonia

Esrafilzadeh, Dorna

Essani, Karim 
Essers, Marieke A.G.

Estelrich, Joan

Estes, Jacob D.

Esteves, Ana Cristina

Estevez, Ana

Etebari, Kayvan

Etienne, Lucie

Etnier, Jennifer L.

Euler, Gerhild

Evangelisti, Edouard

Evangelou, Nikos

Evans, David

Evans, Joseph L.

Evans, Malkanthi

Eve, David J.

Evensen, Øystein

Everts, Helen

Every-Palmer, Susanna

Eyers, Claire

Ezendam, Janine

Ezura, Yoichi

Fabiani, Roberto

Fabregat, Isabel

Facchini, Gaetano

Fadl, Amin

Faehling, M.

Fagerstedt, Kurt

Fahraeus, Robin

Faísca, Patrícia

Falk, Heinz

Falk, Roni T.

Falone, Stefano

Fan, Fengjuan

Fan, Xiucheng

Fañanas, Francisco J.

Fang, Bai

Fang, Jianwen

Fang, Ye

Fanucci, Gail E.

Farber, Steven Arthur

Farinos, H. Merle

Faroni, Alessandro

Farrow, Kathryn

Faul, Christian

Favas, Paulo

Fedder, Jens

Fedele, Francesco

Fedorova, Olga V.

Fei, Likun

Feige, Jerome

Feinstein, Paul

Felder-Schmittbuhl, M.-P.
Felger, J.C.

Feliers, Denis

Feng, Yibin

Feng, Yu

Feng, Z. Vivian

Feo, Francesco

Feraco, Alessandra

Feresin, Rafaela G.

Ferlizza, Enea

Fernandes, Eduarda

Fernández Robredo, Patricia

Fernández, Francisco J.

Fernández, Lucía

Fernandez, Michael

Fernandez-Botran, Rafael

Fernández-García, M. Isabel

Fernandez-Garcia, Marta

Fernandez-Lafuente, Roberto

Fernandez-Lima, Francisco

Fernandez-Palomo, Cristian

Fernandez-Pozo, Noe

Fernández-Ruiz, Javier

Fernie, Alisdair

Ferrand, Jonathan

Ferrandino, Ida

Ferrante, Antonio

Ferraresso, Mariano

Ferrero, Dario

Ferreti, Claudio

Ferretti, Claudio

Ferretti, Elisabetta

Ferrieres, Vincent

Ferrio, J.P.

Ferroni, Patrizia

Fields, Gregg B.

Filella, Xavier

Filep, János G.

Filigheddu, Nicoletta

Filosto, Massimiliano

Fine, James Burke

Finelli, Carmine

Finer, Yoav

Finkelstein, Jacob N.

Fiocco, Daniela

Fiona, Lynch

Fioravanti, Antonella

Fiorenza, Maria Teresa

Fischer, Keelan

Fischer, Silvia

Fisher, Scott

Fiume, Giuseppe

Flamant, Quentin
Flanagan, Sarah P.

Flematti, Gavin R.

Flex, Andrea

Florczyk, Stephen J.

Florea, Ana-Maria

Flores, Francisco B.

Florio, Tullio

Flower, Andrew

Flynn, Aidan

Flynn, Charles R.

Foan, Chin Chiew

Foderà, Vito

Fogarty, Gerald

Fogdell-Hahn, Anna

Fogolari, Federico

Folco, Hernan Diego

Fong, Wye-Khay

Fontalba-Navas, Andres

Fontana, Robert J

Fontecha, Javier

Forbes-Hernandez, T.Y.

Forini, Francesca

Forlemu, Neville

Formisano, Pietro

Formosinho, Sebastião J.

Forn-Cuni, Gabriel

Forner, Stefânia

Foro, Palmira

Forrest, Lucy

Fort, Patrice

Forton, F.M.N.

Fortunato, Orazio

Fossdal, Carl Gunnar

Foster, David

Foster, Kenneth R.

Fournier, Claudia

Fournier, Pierre-Edouard

Fox, Susan

Fozo, Elizabeth

Fracanzani, Anna Ludovica

Fraccarollo, Daniela

Fradley, Michael G.

Frago, Enric

Fragouli, Despina

Francesca, Mangialasche

Franceschi, Pietro

Francino, Pilar

Francis, Heather

Franco, Aime T

Franco, Diego

Franco, Rodrigo

Frank, Stephan 
Franken, Philipp

Franzke, Claus-Werner

Fraser, Justin F.

Freedman, Bruce D.

Freeman, Jennifer L.

Freije, José M.P.

Freise, Christian

Freking, Brad

French, Lars

Frenea-Robin, Marie

Fresco, Paula

Freundt, Eric C.

Friedlander, Terence W.

Froeyen, Matheus

Fröhlich, Eleonore

Frost, Sofia

Fry, Jessica L.

$\mathrm{Fu}$, Jidong

$\mathrm{Fu}$, Shih-Feng

Fuchs, Bruno

Fugazzola, Laura

Fujii, Hirofumi

Fujii, Takao

Fujii, Takeshi

Fujimori, Ko

Fujita, Masayuki

Fujita, Yoshio

Fujitsuka, Naoki

Fujiwara, Ryoichi

Fujiwara, Shin-ichi

Fukuda, Takeshi

Fukuhara, Ryoji

Fukunaga, Kenji

Fukunaga, Kohji

Fukunishi, Yoshifumi

Fukushima, Atsushi

Fukushima, Tsuyoshi

Fulkerson, Patricia C.

Fuller, Bryan

Fullston, Tod

Fulton, Amy M.

Fumihiko, Katakura

Funaba, Masayuki

Fürnsinn, Clemens

Furue, Masutaka

Furuichi, Kengo

Fuselli, Fabio

Fuso, Andrea

Fuster, Daniel

Gaborit, B.

Gabriella, Lindgren

Gaerstel, Matthias
Gafencu, Anca

Gailani, Gaffar

Gailhouste, Luc

Gajofatto, Alberto

Gajula, Rajendra

Gal, A.

Galanakis, Charis M.

Galdiero, Stefania

Gales, Amandine

Galileo, Deni S.

Gall, Henning

Gallardo, Rodrigo A.

Galli, Dominique M.

Gallicchio, Emilio

Gallie, Daniel R.

Gallo, Linda

Galus, Sabina

Galván, Ismael

Gambarotta, Giovanna

Gambino, G.

Gams, Walter

Gan, R.Y.

Ganea, D.

Ganguli-Indra, Gitali

Ganini, Douglas

Gantenbein, Benjamin

Gao, P.

Gao, Xue

Gao, Zhiqiang

Garcia, Michael

García-Jimenez, Iker

Garcia-Redondo, Ana Belen

Gard, Paul

Garinis, George

Garner, Bianca

Garrett, Scott

Garrity, Deborah

Gasparrini, Massimiliano

Gasser, Christoph

Gatti, Laura

Gaudenzio, Nicolas

Gaudet, Andrew

Gavaia, P.J.

Gavira, José A.

Gavish, Moshe

Gazouli, Maria

$\mathrm{Ge}$, Xiaodong

Gee, Christine E

Gehrs, Karen M.

Gekle, Michael

Geldof, Lore

Gelpi, Josep Lluis
Gémes, Katalin

Genetos, Damian

Genji, Imokawa

Genovese, Giuseppa

Gensel, John C.

Gentile, Piergiorgio

Georgakilas, Alexandros G.

George, Graham

George, Varghese

Georgiev, Vasil

Geraci, Fabiana

Gerardi, Carmela

Gerber, Leonard E.

Gerdol, Marco

Gerhard, Markus

Gericke, Martin

Gerothanassis, Ioannis P.

Gershburg, E.

Geso, Moshi

Gewirtz, David

Ghaleh, Bijan

Ghandhi, Shanaz Adi

Gharbia, Saheer E.

Ghavami, Saeid

Ghia, Jean-Eric

Ghosh, Sujoy

Giaginis, Constantinos

Giampieri, Francesca

Gianferrara, Teresa

Giannakourou, Maria C.

Giannattasio, Sergio

Giannenas, Ilias

Giannotti, Marina Inés

Giansanti, Francesco

Giblin, Frank J.

Gibson, Gregory

Gibson, Joshua

Gibson, Susan I.

Gieling, Roben G.

Gieras, Anna

Gilbert, Nick

Gilles, Christine

Gil-Mohapel, Joana M.

Gilmore, Reid

Gilmour, Susan

Gilson, Michael

Gimeno, M. Concepción

Ginty, Fiona

Giordano, Antonio

Giorgio, Ivan

Girgis, Reda E.

Giron, David 
Gismondi, Angelo

Giuliano, Michela

Giuseppe, Zampella

Glading, Angela J.

Glauser, Gaetan

Glavaski-Joksimovic,

Aleksandra

Glazer, Lilah

Glyan'ko, A.K.

Gobbo, Olivero

Goberdhan, Deborah C.I.

Goedert, James

Gohar, Eman

Gohda, Eiichi

Gohla, Antje

Göhre, Vera

Goldberg, Erv

Goldgar, David

Golding, Michael C.

Goldoni, Matteo

Goletti, Delia

Gollahon, Lauren

Goltsov, Alexey

Gomes, Ana P.

Gomes, Maria Salomé

Gómez, Irene Crespo

Gomez, Nidia

Gómez-Florit, Manuel

Gomez-Sanchez, Elise

Gomis, Roger R.

Gonçalves, M. Sameiro T.

Gonçalves, Olivier

Gonda, Thomas J.

Gondhalekar, Ameya D.

Gondi, Christopher S.

Gong, Fade

Góngora-Castillo, Elsa

Gonzales, Cara B.

Gonzalez, Joaquin

Gonzalez, Patrice

Gonzalez, Roser

González-Aguilar, Gustavo

González-Bosch, Carmen

Gonzalez-Conejero, Rocio

Gonzalez-Reyes, Luis E.

González-Rodríguez, M.-L.

González-Sánchez, M.I.

Goodacre, Sara

Goodman, Richard

Goossens, Nicolas

Gopalakrishnan, Singaram

Gorczynski, Paul
Gordon, Donna

Gorgoulis, Vassilis G.

Gorman, Shelley

Gorodetsky, Raphael

Gosetti, Fabio

Gossage, R.A.

Goto, Kazuo

Goto, Yuki

Goua, Marie

Gougelet, Angélique

Goulielmos, George N.

Govaere, Olivier

Gowans, Eric

Gozes, Illana

Graber, Judith

Gradziel, Tom

Graffelman, Jan

Graham, Julie

Graier, Wolfgang

Graille, Marc

Grammatikos, Georgios

Granato, Daniel

Granot, David

Granucci, Francesca

Grãos, Mário

Grapputo, Alessandro

Grasa, Laura

Grases, Felix

Grassl, Guntram A.

Gratzinger, Dita

Gray, Christopher

Greenberger, Joel S.

Greene, Catherine M.

Greene, Lesley H.

Greene, Michael J.

Greenman, John

Greenwood, Michael T.

Gregory, Karen J.

Gregory, Simon G.

Grether-Beck, Susanne

Griffin, William

Griffis, John

Griffith, Kevin

Griffiths, Bryan

Griffiths, Leigh G.

Gril, Brunilde

Grilli, Alfredo

Grimes, Pearl

Grimholt, Unni

Grimm, Elizabeth A.

Grimpe, Barbara

Grobe, Justin L.
Groenendyk, Jody

Grogan, Gideon

Gropman, Andrea L.

Grosche, Antje

Gross, Gideon

Grossini, Elena

Grosso, Clara

Grotta, James C.

Grubman, Alexandra

Gruell, Henning

Grumet, Rebecca

Grzebelus, E.

$\mathrm{Gu}$, Feng

$\mathrm{Gu}$, Haiwei

$\mathrm{Gu}$, Jianguo

Gu, Wenyi

Gualtieri, Maurizio

Guan, Xin

Guarino, Francesco

Gubler, Frank

Gude, Veera Gnaneswar

Guerini, Franca R.

Guerlesquin, Francoise

Guerrero, Pedo

Guerrero-Preston, Rafael

Guerrieri, Emilio

Gugliucci, Alejandro

Guhathakurta, Subhrangshu

Guidi, Lucia

Guignabert, Christophe

Guillou, Hervé

Guilpain, Philippe

Gundogdu, Ozan

Guo, Fenghai

Guo, Lei

Guo, Yan

Gupta, Ankit

Gupta, Prachi

Gupta, Shashi

Gupta, Vijai Kumar

Gupta, Vivek K.

Gupta-Rossi, Neetu

Gürdeniz, Gözde

Gurevich, Vsevolod V.

Gururani, Mayank

Gutiérrez Martín, Santiago

Gutiérrez-Juárez, Roger

Gutschner, Tony

Guyeux, Christophe

Guzman-Puyol, Susana

Guzzo, Flavia

Haaparanta-Solin, Merja 
Haase, Hajo

Häder, Donat P.

Hadjiargyrou, Michael

Hadjikakou, Sotiris K.

Ha-Duong, Nguyet-Thanh

Hadwiger, Lee

Hafizi, Sassan

Hagenbuch, Bruno

Haglund, Lisbet

Hagstrom, Stephanie A.

Hahnel, Sebastian

Haider, Lukas

Haider, Norbert

Hains, David

Halberstadt, Adam L.

Halbwirth, Heidi

Hall, Carolyn S.

Hall, Rebecca

Halterman, Dennis

Halushka, Marc

Hamaguchi, Masahide

Hamamura, K.

Hamanishi, Junzo

Hamilton, James P.

Hamilton, Paul

Hamilton, Stuart T.

Hammer, Suntrea

Hammond, John

Hamwieh, Aladdin

Han, Dong Wook

Han, Quan-Bin

Han, Sang-Bae

Han, Yeon S.

Hanaoka, Mitsumasa

Hanaor, Dorian

Hanash, Alan M.

Hancock, Rob

Handa, Osamu

Hankey, Pamela

Hano, Christophe

Hanus, Jakub

Happel, Austin

Happel, Patrick

Harashima, Nanae

Haraszthy, Violet I.

Harauz, George

Hardeland, Rudiger

Harding, Ian

Hardingham, Jennifer E.

Hardman, W. Elaine

Harduin-Lepers, Anne

Hardy, John
Hare, Joshua M.

Hargrove, Mark

Häring, Hans-Ulrich

Harmsen, Hermie J.M.

Haro, Diego

Harpke, Dörte

Harris, Andrew R.

Harris, Jennifer W.

Harris, L.K.

Harris, Philip

Harris, Randall E.

Harrison, Lisa M.

Harroun, Thad

Harscoat-Schiavo, Christelle

Hartmann-Petersen, Rasmus

Harty, Ronald

Harvey, Ruth

Hasegawa, Koichi

Hashimoto, Masayuki

Häsler, Robert

Hassan, Mohamed

Hassouna, Rim

Hata, Aaron

Hatano, Tsutomu

Hatfield, Ronald D.

Hattori, Toshio

Hattori, Yuichi

Hatzopoulos, Polydefkis

Haukaas, Tonje

Haukka, Kaisa

Hausner, Georg

Hautbergue, Guillaume

Hawes, T.C.

Hawinkels, Lukas

Hayakawa, Tohru

Hayward, S. Diane

Hazane-Puch, Florence

Hazell, Alan S.

Hazlehurst, Jonathan

$\mathrm{He}$, Xiao

He, Xiaojuan

He, Zhen

Hearn, Michael J.

Hebbard, L.

Hebelstrup, Kim H.

Hébert, Terry

Hecker, Michael

Hedemann, Mette Skou

Hedfalk, Kristina

Hedhammar, My

Hedley, Peter

Heegaard, Niels H.H.
Hefferon, Kathleen Laura

Hegazi, Refaat A.

Hegyi, Eszter

Hegyi, Péter

Hehlgans, Stephanie

Heianza, Yoriko

Heidari, Mohammad

Heideman, Warren

Heider, Dominik

Heise, Henrike

Helle, Francois

Heller, E. Dan

Hellwig, Michael

Helm, Richard

Hemley, Sarah Jane

Hemmer-Hansen, Jakob

Henke, Andreas

Heras, Joseph

Herbst, Andreas

Hernández-Alcoceba, Rubén

Hernández-Castellano, L.E.

Hernández-Rodríguez, César

Hernández-Rodríguez, José

Herr, Deron Raymond

Herrero, María Jesús

Hertz, Daniel L.

Hervás-Pérez, J.P.

Hess, Angela

Hetman, Michal

Hettiarachchi, D.S.

Hettinga, Kasper

Hewett, James A.

Heyn, Patricia

Hiblot, Julien

Hicks, Alexander

Hicks, Glenn R.

Hidalgo-Cantrabana, Claudio

Hider, Robert

Higashi, Tomomi

Higgins, Claire

Higuchi, Akon

Hildebrand, Mark

Hildt, Eberhard

Hill, William G.

Hiller, Monika

Hiltermann, T. Jeroen N.

Hiltner, Jana Katharina

Hilz, Max

Hingorani, Dina

Hiraga, Toru

Hirakawa, Hidehiko

Hirano, Katsuya 
Hirano, Shigeru

Hirasawa, Noriyasu

Hirasawa, Takashi

Hiroaki, Fujii

Hirohashi, Yoshihiko

Hirotsune, Shinji

Hirsch, Pierre

Hleap, Jose Sergio

Ho, Roger Chun-Man

Hoeijmakers, Janneke G.J.

Hoemann, Caroline D.

Hoene, Miriam

Hoffmann, Georg

Höfinger, Siegfried

Hofmann, Johann

Hofmann, Thomas G.

Hojjat-Farsangi, Mohammad

Holder, Alvin

Holland, Gregory

Holliday, L. Shannon

Höllig, Anke

Hollman, Peter C.H.

Hollomon, Mario G.

Holmes, Dawn E.

Holmes, Greg

Holt, Scott M.

Höltke, Carsten

Holton, Therese

Holzinger, Michael

Holzmann, Nicole

Homem, Rafael

Hondan, Tomoyuki

Hong, Kyeong-Man

Hong, Yeong Ho

Hong, Yonggeun

Hong, Yunhan

Hönicka, Markus

Honoré, Patrick M.

Hooper, Antony Mark

Hordvik, Ivar

Horsefield, Susanna

Horvath, David P.

Hoshi, Akihiko

Hotta, Akitsu

Hottiger, Michael

Hou, Chien-Wei

Hou, Harvey

Hou, Yueh-Ju

Houen, Gunnar

Houghton, David

Houston, Kevin D.

Houston, Mark C.
Houston, Ross

Hribal, Marta

Hrobárik, Peter

Hsia, Shih-Min

Hsiao, George

Hsieh, Nan-Hung

Hsieh, Pei-Wen

Hsieh, Yi-Hsien

Hsin, Kun-Yi

Hsu, Daniel

Hsu, Li-Sung

Hsu, Todd

$\mathrm{Hu}$, Michael Sung-Min

$\mathrm{Hu}$, Ning

$\mathrm{Hu}$, Shao-Yang

$\mathrm{Hu}$, Yinling

$\mathrm{Hu}, \mathrm{Yu}$

Huang, A-Mei

Huang, Chi-Chang

Huang, Chih-Ling

Huang, Chiu-Jung

Huang, Genin Gary

Huang, Hao

Huang, Hsuang-Ting

Huang, Jian

Huang, Jianguo

Huang, Renhua

Huang, Shih-Yi

Huang, Tzou-Chi

Huang, Wen-Chin

Huang, Wen-Lii

Huang, Wenlin

Huang, Yuting

Huang, Zhan-Peng

Huang, Zhi

Huangfu, Danwei

Huchzermeyer, Bernhard

Huelsen, Tim

Huen, Michael Shing Yan

Huertas-Pérez, José Fernando

Hughes, Timothy C.

Huh, Jin Hoe

Huhn, Ragnar

Hui, Ye

Huleihel, Mahmoud

Hulten, Kristina G.

Hung, Jan-Jong

Hunsicker-Wang, Laura M.

Hunt, Piper Reid

Hunter, Wayne B.

Huppi, Konrad

Huq, Laila
Hur, Wonhee

Hura, Tomasz

Hurst, Douglas R.

Hursthouse, Andrew

Husnjak, Koraljka

Hüttemann, Maik

Hwang, Bang Yeon

Hwang, Dae Youn

Hwang, Eun Sook

Hwang, Ming-Jing

Hwang, William Ying Khee

Hwang, Woo Suk

Hyun, Jin Won

Hyun, Sang-Hwan

Iacobellis, Gianluca

Iadarola, Paolo

Iadevaia, Valentina

Iannuccelli, Valentina

Iasevoli, Felice

Ibarra Molero, Beatriz

Ibrahim, Hussameldin

Ibrahim, Toni

Ichikawa, Hiroshi

Ichimura, Hiroshi

Iebba, Valerio

Ientile, Riccardo

Iglesias, Emilia

Iguchi, A.

Ihalainen, Teemu O.

Ihle, Andreas

Iida, Hiroshi

Iinuma, Hisae

Ikai, Tomoyuki

Ikeda, Hajime

Ikeda, Masahiro

Ikeshima-Kataoka, Hiroko

Ilardi, Vincenza

Illa, Miriam

Imai, Kazushi

Imajoh, Masayuki

Imig, Jochen

Immel, Françoise

Inaba, Hiroshi

Inagaki, Naoki

Indo, Hiroko P.

Indriolo, Emily

Inman, Sharon

Inokuchi, Tsutomu

Inoue, Atsuko

Inoue, G.

Inoue, Yasumichi

Insel, Kathleen C. 
Inselman, Amy

Inta, Dragos

Inui, Hideyuki

Inui, Masafumi

Inuzuka, Hiroyuki

Iolanda, Francolini

Iorio, Egidio

Iorio, Raffaele

Iorizzo, Massimo

Iraci, Nunzio

Irakli, Maria

Irato, Paola

Irina, Moreira

Irjala, Heikki

Isaji, Shuji

Isemura, Mamoru

Ishibashi, Kenichi

Ishii, Isao

Ishikawa, Yasuko

Ishitsuka, Yoichi

Ishizaki, Takuma

Islam, M. Nurul

Islam, Md Soriful

Isman, Murray

Isokpehi, Raphael

Ito, Shigeki

Ito, Shosuke

Ito, Yasuhiro

Itoh, Kazuyuki

Itoh, Masayuki

Itoh, Toshiyuki

Itoh, Yoshifumi

Itou, Junji

Ivankin, Andrey

Ivanov, Alexander

Iwaizumi, Masakazu G

Iwamori, Masao

Iwamoto, Sadahiko

Iwashima, Yoshio

Izquierdo-Useros, Nuria

Izumi, Kouji

Izumi, Namiki

Izumiya, Yoshihiro

Jack, Thomas

Jackson, Christopher

Jackson, Miriam T.

Jackson, Stephen

Jacob, Christoph R.

Jacob, Francis

Jacobsen, Elisabeth Egholm

Jacot, William

Jacquin, Lisa
Jaeschke, Hartmut

Jagerovic, Nadine

Jagiello, Karolina

Jaimovich, Enrique

Jaiswal, Ashvin R

Jaiswal, J.K.

Jakob, Stephan M.

Jakočiūnas, Tadas

Jakowski, Joseph D.

Jalkanen, Sirpa

James, Euan

James, Nicholas G.

Jan, Fuh-Jyh

Jang, Ik-Soon

Jang, Jer-Huan

Jang, Kiseok

Jang, Seonghoe

Jang, Wooyoung

Jann, Michael W.

Jansen, Eugen

Janssen, Arne

Jarausch, Wolfgang

Jarret, Robert L.

Järvenpää, Eila P.

Jarvis, Gary A.

Jat, Parmjit S.

Jauch, Ralf

Jaumot, Joaquim

Jawien, Jacek

Jayaraman, Dhileepkumar

Jayasinghe, Suwan

Jay-Gerin, Jean-Paul

Jay-Russell, Michele

Jazirehi, Ali

Jendelova, Pavla

Jennings, Lance $C$.

Jensen, Eric

Jensen, L.H.

Jensen, Randy L.

Jensen, Thomas

Jeong, Byoung Ryong

Jeong, Daewon

Jeong, Jeeyon

Jeong, Keun-Yeong

Jeong, Seon-Yong

Jeong, Won-Joong

Jeong, Woo-Sik

Jeschke, Udo

Jesionowski, Teofil

Jetten, Anton

Jeyaseelan, Kandiah

Jeyasekharan, Anand
Jha, Amit

Jheon, Sanghoon

Ji, Debin

Jia, Baolei

Jia, Lei

Jiang, Mei

Jiang, Qing

Jiang, Shu-Ye

Jiang, Wei

Jiang, Xiaohua

Jickling, Glen

Jiménez-Araujo, Ana

Jiménez-Cervantes, Celia

Jin, Dong-Hoon

Jin, Ge

Jin, Huajie

Jin, Li

Jin, Xin

Jing, Fuyuan

Job, Dominique

Joensuu, Jussi J.

Joglekar, Madhura

John, Rohan

Johnson, Arlen W.

Johnson, Gregory A.

Johnson, Jason L.

Johnson, Paul D.

Johnson, Thomas V.

Johnston, G. Patricia

Johnston, Michael

Joles, Jaap

Jolkkonen, Jukka

Jones, Angus

Jones, Brian

Jones, Davy

Jones, Huw

Jones, John

Jones, Rachel A.

Jones, T. Bucky

Jonnalagadda, Subash C.

Joo, Choun-Ki

Jordao, Antonio M.

Jörg, Frederike

Jørgensen, Anders Palmstrøm

Jørgensen, Malene

Jose, Pedro A.

Joseph, Shannon

Joshi, Bharat

Jouanneau, Sulivan

Joubert, Michael

Jouneau, Alice

Joußen, Nicole 
Jovanović, Aleksandar

Joven, Jorge

Jover, Ramiro

Joyce, Susan A.

Juang, Horng-Heng

Juarranz, Ángeles

Jujić, Amra

Juliano, Claudia

Julier, Bernadette

Jun, Ho-Wook

Jung, Eunsun

Jung, Hyungtaek

Jung, Klaus

Jung, Seunho

Jung, Sophie

Jung, Yong Woo

Jung, Young Mee

Junker, Kerstin

Jurjus, Rosalyn A.

Juszczak, Lesław

Jyh-Fei, Liao

Kabbani, Toufik A.

Kaczor, Agnieszka

Kadib, Abdelkrim El

Kadokawa, Jun-ichi

Kaeffer, Bertrand

Kageyama, Yukio

Kahn, Michael

Kai, Mihoko

Kaira, Kyoichi

Kaji, Hiroshi

Kaji, Toshiyuki

Kajiya, Hiroshi

Kakinuma, Sei

Kakinuma, Yoshihiko

Kakisis, John D.

Kako, Tetsuya

Kakulas, Foteini

Kalendar, Ruslan

Kalfakakou, Vasiliki

Kalinin, Stanislav

Kaller, Markus

Kallunki, Tuula

Kalogiannis, Konstantinos G.

Kalogirou, Charis

Kamagata, Kiyoto

Kamal, Abu Hena Mostafa

Kambe, Taiho

Kamihira, Masamichi

Kamiloglu, Senem

Kaminskyj, Susan

Kamioka, Hiroshi
Kamitani, Shigeki

Kamiya, Hideki

Kamiya, Katsumasa

Kamp, Christel

Kamp, David W.

Kamphuis, Lars G.

Kamunde, Collins

Kan, Casina

Kanaoka, Masahiro

Kane, Maureen A.

Kang, Bum-Yong

Kang, Byoung-Cheorl

Kang, Chen

Kang, Chulhun

Kang, Hunseung

Kang, Jae Seung

Kang, Jaeku

Kang, Jeehoon

Kang, Jonghoon

Kang, Lei

Kang, Min-Jung

Kang, Sang Sun

Kang, Sungmin

Kang, Taek Won

Kang, Wei

Kang, Wonku

Kango-Singh, Madhuri

Kanvil, Sadia

Kanzaki, Hiroyuki

Kao, Erl-Shyh

Kapfhammer, Josef

Kaplan, Barry B.

Kappen, Jasper H.

Karakasidis, T.E.

Kararigas, Georgios

Kärenlampi, Sirpa

Karginov, Fedor

Karkhanis, Vrajesh

Karki, Rajendra

Karlic, Heidrun

Karlsson, Oskar

Kårlund, Anna

Karmakar, Partha

Karras, S.N.

Kasahara, Hideko

Kaschina, Elena

Kashman, Yoel

Kashuba, Elena

Kasoju, Naresh

Kassiri, Zamaneh

Kasten-Jolly, Jane

Katam, Ramesh
Kataoka, Naoyuki

Katapodis, Petros

Katayama, Shigeru

Kato, Hirohito

Kato, Ken

Kato, Koichi

Kato, Kumiko

Kato, Souichiro

Kato, Takamitsu A.

Katoh, Masaru

Katoh, Shigeki

Katsantonis, Dimitrios

Katsila, Theodora

Katsoris, Panagiotis

Katsuya, Tomohiro

Kattner, Lars

Katus, Hugo

Kauppinen, Anu

Kaur, Sukhjiwan

Kaur, Tejbeer

Kawabata, Saneyuki

Kawada, Kenji

Kawada, Manabu

Kawahara, Ei

Kawai, Vivian K.

Kawakami, Susumu

Kawamata, Shin

Kawamura, Akifumi

Kawamura, Izuru

Kawamura, Kazuhiro

Kawano, Kouichiro

Kawaratani, Hideto

Kawasaki, Ichiro

Kawashima, Makoto

Kawasumi, Koh

Kawauchi, Akihiro

Kayano, Shin-ichi

Kazlauskas, Andrius

Ke, Youqiang

Keep, Richard

Keir, Iain

Kelleher, Fergal C.

Kellenberger, Stephan

Keller, Amy Celeste

Keller, Evan T.

Keller, Jesse J.

Kelley, Thomas

Kellner, Franziska

Kellner, Ronny

Kelly, Clive

Keltanen, Terhi

Kelts, Jessica 
Kemp, Kevin

Kennedy, Patrick

Kent, Robert M.

Kersten, Sander

Keyaerts, Marleen

Khadka, Manoj

Khaliulin, Igor

Khan, Imran

Khan, Mushfiquddin

Khanna, Nidhi

Khazaie, Khashayarsha

Khoo, Bee Luan

Khoobchandani, Menka

Khozin-Goldberg, Inna

Khuder, Sadik A.

Khurshid, Zohaib

Kibriya, Muhammad G.

Kiddle, Guy

Kidoaki, Satoru

Kiesslich, Tobias

Kietzmann, Thomas

Kihm, Lars P.

Kikuchi, Eiji

Kim, Byeongil

Kim, Chaerin

Kim, Gi Jin

Kim, Gloria J.

Kim, Hoon

Kim, Hye Ryoun

Kim, Hyun Ah

Kim, Hyung Sik

Kim, Il-Man

Kim, Jae Young

Kim, Jin

Kim, Jin Moon

Kim, Jin-Bom

Kim, Jin-Wook

Kim, Jong Bum

Kim, Joungmok

Kim, Julian

Kim, Ki-Hong

Kim, Kyung-Min

Kim, Kyung-Su

Kim, Na-Hyung

Kim, Sang-We

Kim, Seok Jin

Kim, Soo-Un

Kim, Suk

Kim, Sung-Hoon

Kim, Sung-Whan

Kim, Tae Il

Kim, Won Ho
Kim, Woo-Jae

Kim, Yong Soo

Kim, Yong-June

Kim, Youngsoo

Kim, Yun Kyung

Kim, Yun-Bae

Kimura, Hideto

Kimura, Ikuko

Kimura, Tadashi

Kimura, Tomoki

King, Bonnie L.

King, Mary Lou

King, Mike

Kinghorn, Kerri J.

Kinnula, Hanna

Kinoshita, Takayoshi

Kipp, Anna Patricia

Kirby, Ralph

Kirsch, Thorsten

Kirschning, Carsten J.

Kishimoto, Yoshimi

Kishimoto-Yamada, Keiko

Kisiel, John B.

Kissling, Jonathan

Kitamura, Naoki

Kitatani, Kazuyuki

Kitchen, Scott G.

Kittner, Jens M.

Kiyama, Ryoiti

Kiyan, Yulia

Kleeff, Jörg

Klegeris, Andis

Klein, Kerstin

Kleinekathöfer, Ulrich

Kleuser, Burkhard

Klimyuk, Victor

Klussmann, Enno

Kluza, Jérome

Knapp, Deborah

Knapp, Stefan

Knecht, Hans

Knight, Christopher

Knight, Jason S.

Kniss, Douglas A.

Knock, Greg A.

Knott, Rachel

Knowles, Margaret A.

Kobayashi, Hiroshi

Kobayashi, Satoru

Kobayashi, Tatsuya

Kobeissy, Firas H.

Kobori, Hiroyuki
Kocarnik, Jonathan M.

Köcher, Thomas

Kocmarek, Andrea L.

Koczera, Patrick

Kodama, Daisuke

Koduru, Janardhan Reddy

Koea, Jonathan

Koga, Fumitaka

Kogel, Karl-Heinz

Koh, Hyongjong

Koh, Timothy

Kohen, Ron

Kohno, Jun-Ya

Kohri, Michinari

Koistinen, Hannu

Kojima, Chie

Kojima, Masami

Kojima-Yuasa, Akiko

Kojo, Shosuke

Kokai, Lauren E.

Kolasinski, Kurt W.

Kolassa, Iris-Tatjana Kolassa

Koller, Martin

Kollmar, Otto

Komatsu, David

Komatsu, Shuhei

Komis, George

Komohara, Yoshihiro

Komoike, Yuta

Kondo, Eisaku

Kondo, Hidehiro

Kondo, Toshihiro

Kong, Won-Sik

Konig, Heiko

Koninckx, Philippe R.

Konishi, Hirotaka

Konkol, Yvonne

Konno, Katsuhiro

Kono, Hiroshi

Kooijman, Ron

Kooiman, Klazina

Koon, Hon Wai

Koonen, Debby P.Y.

Kootala, Sujit

Kopecki, Zlatko

Kopel, Pavel

Koperek, Oskar

Koppara, Tobias

Korbelik, Mladen

Kornek, Miroslaw

Korsching, Eberhard

Kosova, Klara 
Kostakis, Ioannis

Kostin, Sawa

Kota, Satya

Kotloski, Robert

Kotsaki, Antigone

Kotsikorou, Evangelia

Kouadio, James Halbin

Kouretas, Dimitrios

Kourist, Robert

Kourkoumelis, Nikolaos

Kourkoutas, Yiannis

Kousoulas, Konstantin G.

Kouvelis, Vassili N.

Kowalski, Konrad

Kox, M.

Koyama, Yu

Kozlevcar, Bojan

Kozliak, Evguenii I.

Krackhardt, Angela

Kramer, Phillip

Kratochwil, Claudius

Kratochwil, Clemens

Krautwald, Stefan

Krawczyk, Pawel

Krebs, Michael

Krepkiy, Dmitriy V.

Kretz, Markus

Kreuzwieser, Juergen

Krishnaswamy, Guha

Kristian, Tibor

Krjutškov, Kaarel

Kroncke, Brett M.

Krüger, Marcus

Kruger, Maria J.

Kruyt, Frank A.E.

Krysinska, Karolina

Krzystek-Korpacka, M.

$\mathrm{Ku}$, Seung-Yup

Kuan, Yu-Hsiang

Kuang, Huihui

Kubota, Keiichi

Kubota, Naoto

Kucerik, Jiri

Kucukkal, Tugba

Kukreja, Subhash

Kulak, Nora

Kumada, Takayuki

Kumar, Gaurav

Kumar, Sandeep

Kumar, Sathish

Kumar, Sushil

Kumar, Ujendra
Kumazawa, Yoshinori

Kundu, Aishwarya

Kunnimalaiyaan, M.

Kunz, Wolfram

Kunze, Gotthard

Kuo, Hsing-Chun

Kuo, Ping-Chung

Kuo, Tzong-Fu

Kuo, Ya-Huei

Kupfer, Alexander

Kuppe, Christoph

Kurdowska, Anna K.

Kurepin, Leonid V.

Kurpisz, Maciej

Kurschus, Florian C.

Kurtcuoglu, Vartan

Kurz, Tino

Kusakabe, Makoto

Kusumbe, Anjali

Kuwahara, Keisuke

Kuwata, Keith T.

Kwa, Faith A.A.

Kwakowsky, Andrea

Kweon, Oh-Kyeong

Kwok, Jessica

Kwon, Tae Gyun

Kwon, Tae-Hwan

Kwon, Young-Wan

Kwong, Joseph

Kyprianou, Natasha

Kyzas, George

Laboisse, Christian L.

Lachman, Jaromir

Lachowicz, Joanna Izabela

Lafontaine, Denis L.J.

Laforenza, Umberto

Lahiri, Amitabha

Lahooti, Hooshang

Lai, Christopher W.K.

Lai, Hung-Cheng

Lai, Hung-Ming

Lai, Shih-Wei

Laity, Peter R.

Lam, Kim-Hung

Lamas, José Ramón

Lamb, David

Lamers, Susanna L.

Lan, Lan

Landete, José Maria

Landi, Abdolamir

Landriscina, Matteo

Lane, Hsien-Yuan
Lanekoff, Ingela

Lange, Kathrin M.

Langenhan, Tobias

Lansdon, Eric B.

Lao, T.T.

Laplante, Mathieu

LaPres, John J.

Laranjo, Marta

Larena, Inmaculada

Laroche, Céline Laroche

Larsen, Flemming

Larsen, Paul B.

Larson, Nicholas B.

Larsson, Christer

LaSalle, Janine

Lash, Lawrence

Lassmann, Hans

Lassot, Iréna

Latinovic, Olga

Lattanzi, Wanda

Lau, Kin-Hing William

Lau-Cam, Cesar A.

Launikonis, Bradley

Laurenceau, Emmanuelle

Laurienzo, Paola

Lauzon, Carol R.

Lavandera, Ivan

Laville, Maurice

Lavrik, Inna N.

Lawlor, Kate

Laxton, Ross C.

Lay, Jackson O.

Lazarević, Vladimir

Lazartigues, Eric

Lazzarato, Loretta

Lazzeri, Chiara

Le Hir, Rozenn

Le Questel, Jean-Yves

Le, Minh

Leach, Katie

Leanza, Luigi

Leask, Andrew

Lebaron, Richard

Lebaron, Simon

Lebeck, J.

Lebleu, Bernard

Lebre, Maria C.

Lechel, Andre

Lechpammer, Mirna

Lecureur, Valerie

Ledesma, Maria Dolores

Ledoux, Mark 
Lee, Bee Wah

Lee, Byung C.

Lee, Che-Hsin

Lee, Chi

Lee, Chia-Hung

Lee, Dae Ho

Lee, Dong Hun

Lee, Gabsang

Lee, Gwo-Shu Mary

Lee, Hsiang-Chieh

Lee, Hsin-Chen

Lee, Huei

Lee, Huei-Jane

Lee, Hye Suk

Lee, J.-H.

Lee, Jae-Ho

Lee, Jandee

Lee, Jen-Ai

Lee, Jiyoun

Lee, Juliana Tsz Yan

Lee, Jun Sik

Lee, Keun-Hyeung

Lee, Kin Wah Terence

Lee, Kuo-Kau

Lee, Ming-Fen

Lee, Moo-Seung

Lee, Myung Koo

Lee, Sam W.

Lee, Sang-Han

Lee, Sanghyeob

Lee, Seoul

Lee, Tzong-Shyuan

Lee, Vincent Ws

Lee, Y.-H.

Lee, Yi-Jang

Lefèvre, Thierry

Leffler, Jonatan

Lehmann, Christian

Lehto, Vesa-Pekka

Lei, Benfang

Leigh, Spencer A.

Leitão, Ana

Leite, Claudia

Lemieux, M. Joanne

Lenaerts, Kaatje

Lenaz, Giorgio

Leng, Roger

Lennox, Kim

Lentini, Giovanni

Lenucci, Marcello S

León, Gerardo

Leonard, Stephen
Leong, David Tai

Leonhard, Matthias

Leonhardt, Ralf M.

Leoni, Alberto

Leopold, Jane A.

Lephart, Edwin D.

Leporatti, Stefano

Leppänen, Jenni

Lessard, Christopher

Lesueur, Didier

Leung, Chung-Hang

Leung, Elaine Lai-Han

Leung, Ricky Yuet-Kin

Leuridan, Elke

Levänen, Bettina

Levato, Riccardo

Levi, Moshe

Levine, Andrew J.

Levingstone, Tanya

Levraud, Jean-Pierre

Levy, David N.

Levy, Michael

Levy, Steven

Lewis, James H.

Lewis, Randolph

Li, Dongmei

$\mathrm{Li}, \mathrm{Fu}$

Li, Guoqiang

Li, Hsing-Hui

Li, Jianneng

$\mathrm{Li}$, Jun

$\mathrm{Li}$, Jun-Xu

Li, Kuo-Bin

Li, Lei

Li, Li

Li, Ling

Li, Linlin

Li, Ning

Li, Qiang

Li, Tiangang

$\mathrm{Li}$, Wanlong

Li, Xiaoli

Li, Yang

Li, Yangming

Li, Yefu

Li, Ying-Ji

Li, Yonggang

Liang, Rongguang

Liang, Xue-Hai

Liao, Francesca-Fang

Liao, Jen-Chung

Liao, Lili
Liao, You-Di

Liapi, Charis

Libbrecht, Romain

Liebman, Joel F.

Liebrand, Thomas W.H.

Liechti, Matthias E.

Liew, Woei Chang

Lightfoot, J. Timothy

Ligterink, Wilco

Liguori, C.

Liguori, Maria

Lim, Bora

Lim, Do-Sun

Lim, Edwin

Lim, Eun-Kyung

Lim, Ki-Byung

Lim, Kyung-Min

Lim, Sung-Jig

Limon, Agenor

Lin, Cheng-Wen

Lin, Chi-Chen

Lin, Chiou-Feng

Lin, Guiting

Lin, Ho

Lin, Hui

Lin, Hung Wen

Lin, Hung-Yin

Lin, Kwang-Huei

Lin, Li

Lin, Liang-In

Lin, Liang-Tzung

Lin, Li-Huei

Lin, Ming-Kuem

Lin, Pao-Hwa

Lin, Pei-Hui

Lin, Tsan-Piao

Lin, Yuan-Chung

Lindenmann, Jörg

Lindsay, Laura A.

Lindstrand, Anna

Linert, Wolfgang

Ling, Hui

Ling, Maurice $\mathrm{Ht}$

Lingappan, Krithika

Linnstaedt, Sarah

Lio, Domenico

Liou, Ying-Ming

Lipke, Peter N.

Lippert, Eric

Lips, Katrin S.

Liu, Alvin Y.

Liu, Bin 
Liu, Botao

Liu, C.Y.

Liu, Chao-Lin

Liu, Delong

Liu, Dongxu

Liu, Fan

Liu, Feng

Liu, Jian

Liu, Jinn-Liang

Liu, Jinxuan

Liu, Jun-Jen

Liu, Li

Liu, Qi

Liu, Qing

Liu, Rengyun

Liu, Shengquan

Liu, Shih-An

Liu, Shing-Hwa

Liu, Zhixia

Liu, Ziqing

Lizee, Gregory A.

Lleò, Maria M.

Lo Muzio, Lorenzo

Lo, Hui-Chen

Lo, Shih-Yen

Locatelli, Marcello

Loebel, Antony

Lofrumento, Dario D.

Loizides-Mangold, Ursula

Lokshin, Anna

Lombardi, Paolo

Lonardo, Amedeo

Loo, Jennifer D.

Lood, Christian

Loos, Katja

López-Guerrero, Antonio

Lopez-Llorca, Luis

Lophatananon, Artitaya

Loquet, Antoine

Lora-Tamayo, Jaime

Lorberboum-Galski, Haya

Lord, Janet

Lord, Megan

Lorenz, Kristina

Lorenzini, Antonello

Lorenzo, Óscar

Lorico, Aurelio

Lou, Tsung-Lin

Loudig, Olivier D.

Love-Gregory, Latisha D.

Loveridge, Joel

Lowe, Rohan
Loxham, Matthew

Lu, Chi-Yu

Lu, Hua

Lu, Linchao

Lu, Mei-Chin

Lu, Ming-Wei

Lu, Paul

Lu, Pei-Jung

Lu, Shenzhou

Lu, Shuo

Lu, Yang

Lu, Yen-Shen

Lu, Yongke

Lucacchini, Antonio

Lucarini, Guendalina

Luchetti, MIchele

Luco, Reini F.

Luconi, Michaela

Ludwig, Christian

Luetgehetmann, Marc

Lui, Ed

Lui, Vincent Chi-Hang

Luigi, Scipione

Łukasik, Rafał

Lukaszewski, Adam

Luna, Jonny E. Duque

Lung, Maria Li

Luo, Huacheng

Luongo, Livio

Lupiáñez, José A.

Lupold, Shawn E.

Luque, Francisco

Łuszczek-Trojnar, Ewa

Lutfy, Kabirullah

Lütken, Henrik

Lutz, Jens

Luu, Doan-Trung

Lymperopoulos, Anastasios

Lynd, Nathaniel

Lyng, Heidi

Lyons, Paul E.

Lyons, Russell

Ma, Dik-Lung

Ma, Guojia

Ma, Qing

Ma, Wei

Ma, Xingzhe

Ma, Yan

Ma, Yiyi

Ma, Yongjie

Maccallini, Cristina

Maccari, Giuseppe
Maccari, Rosanna

Macias, Rocio I.R.

Mack, Andreas F.

MacMillan, Freya

MacPherson, Rebecca E.K.

Madesis, Panagiotis

Madhu, Rajesh

Maekawa, Shinya

Magalhães, Júlia M.C.S.

Maggioni, Daniela

Maggioni, Daniele

Magiatis, Prokopios

Magnaldo, Thierry

Magne, David

Mahajan, Sahil

Maher, Pamela

Mahgoub, Amar

Mahimainathan, Lenin

Mahoney, Sara E.

Mahony, Timothy John

Mai, Sabine

Maier, Patrick

Maillard, Ivan

Maioli, Emanuela

Majewski, Michał

Majumder, Mousumi

Mäkelä, Miia R.

Makino, Takeshi

Makishima, Makoto

Malafa, Mokenge

Malamud, Daniel

Malde, Alpesh

Malemud, Charles

Malheiro, Ricardo

Malhotra, Vivek

Malinauskas, Mangirdas

Malkoc, Veysi

Malli, Roland

Mallikaratchy, Prabodhika

Mallipeddi, Prema Latha

Malm, Heli

Malter, James

Manabe, Ichiro

Mandolesi, Georgia

Manetti, Mirko

Manga, Prashiela

Mangiatordi, Giuseppe Felice

Mann, Karen P.

Manninger, Martin

Manor, Danny

Manouchehrinia, Ali

Manrique, Camila 
Mansley, Morag

Mantamadiotis, Theo

Mante, Francis K.

Manthey, John A.

Manuali, Elisabetta

Manzardo, A.M.

Mao, Chuanbin

Mao, Meng

Marabese, Mirko

Marasco, Daniela

Marcal, Helder

Marcel, V.

Marcelli, Augusto

Marcelo, Filipa

Marchesan, Silvia

Marchetti, Philippe

Marchini, Sergio

Marcos, Mercedes

Marcos, Miguel

Marcovici, G.

Margalef, Maria

Margaria, Paolo

Margaria, Valentina

Margaritis, Lukas H.

Maria José, R.L.

Marin, Jose J.G.

Marini, Juan C.

Mariniello, Loredana

Marino, Joseph

Markopoulos, Anastasios K.

Markopoulou, Olga

Marmiroli, Marta

Marmiroli, Nelson

Marondedze, Claudius

Maroni, Paola

Maroon, Joseph

Maroteaux, Luc

Marsano, Francesco

Marsik, Petr

Marston, Denise A.

Martens, John W.M.

Martí, Sérgio

Martienssen, Robert A.

Martin, Alastair

Martin, Finian

Martin, Jessica

Martin, Luc J.

Martin, Sophie

Martín-Aragón, Sagrario

Martinelli, Paola

Martinez, Manuel

Martinez-Arguelles, D.B.
Martínez-Balibrea, Eva

Martínez-Campa, Carlos

Martinez-Castelao, Alberto

Martínez-Chantar, María Luz

Martínez-Mayorga, Karina

Martínez-Rivas, J.M.

Martinez-Sanchez, Aida

Martini, Claudia

Martini, Xavier

Martino, Sabata

Martinotti, Giovanni

Martins, Joana T.

Maruf, Abdullah Al

Maruyama, Kei

Maruyama, Kyonoshin

Marverti, Gaetano

Marzban, Hassan

Mas, Eric

Masella, Roberta

Mashima, Ryuichi

Masi, Antonio

Mason, Rebecca

Masotti, Andrea

Massie, Charlie

Masters, Colin L.

Mastrangelo, Anna M.

Mastrocola, Raffaella

Masuda, Kiyoshi

Masui, Kenta

Masuo, Kazuko

Mate, Sebastian

Mathura, Venkatarajan S.

Matin, Abdul

Matoba, Nobuyuki

Matos, Ana Rita

Matsuda, Hisashi

Matsufuji, Hiroshi

Matsugi, Masato

Matsugo, Seiichi

Matsui, Kenji

Matsui, Mary S.

Matsui-Yuasa, Isao

Matsumoto, Ken-ichiro

Matsumoto, Yasuhiko

Matsuoka, Daisuke

Matsuoka, Makoto

Matsushita, Takehiko

Matsuura, Bunzo

Matsuura, Kazunori

Matsuzaki, Toshiyuki

Matta, Csaba

Matta, Jamie L.
Mattei, Fabrizio

Matthaei, Hanno

Matthews, Ben

Mattijssen, Frits

Mattner, Jochen

Maugeri-Saccà, Marcello

Maupin, C. Mark

Mauriello, Gianluigi

Mavrogonatou, Eleni

Mavromoustakos, Thomas

May, Philip

Mayhan, William G.

Maynard, Desmond J.

Mazzoleni, Stefano

McAuley, Julie L.

McCall, Kimberly

McCallum, Jason L.

McCamant, David

McCarty, Nami McCarty

McCaughan, G.W.

McCoard, S.A.

McConathy, Jonathan

McCullough, Amanda

Mccully, Kilmer S.

McDaid, Hayley

McDaneld, Tara G.

Mcdonald, Courtney

McDonald, Karen

McDougall, Gordon

McElroy, Kyle

McFarlane, Heather E.

McGarrigle, Eoghan M.

McGarry, Roisin C.

McGhee, James D.

McGrail, Maura

McGrath, Scott

Mcgrew, Michael J.

McIntyre, Jenifer K.

McMahon, Lawrence P.

McMillin, Matthew

Mcmorrow, Tara

McMurray, Fiona

McPhee, Jamie

Mcsharry, Charles

Meade, Mark E.

Mécheri, Salaheddine

Mechler, Adam

Medici, Serenella

Medin, Jeffrey A.

Mehaffy, Carolina

Mehra, Reena

Mehrabi, Arianeb 
Mei, Nan

Meier, Carola

Meijer, H.J.G.

Meijón, Mónica

Meldolesi, Jacopo

Melillo, Rosa Marina

Mellon, Isabel

Melnik, Bodo C.

Memarzadeh, Kaveh

Memon, M.A.

Ménard-Moyon, Cécilia

Menassa, Rima

Mende, Susann

Mendes-Ferreira, Alexandra

Méndez-Ardoy, Alejandro

Méndez-Sánchez, Nahum

Menendez Ramos, J.C.

Menendez, Daniel

Menendez, Esther

Meng, Xiangbing

Menkhorst, Ellen

Menu, Eline

Menzies, Robert I.

Mercader, Josep

Mercati, Francesco

Mercer, Carol

Mercuri, Nicola Biagio

Merkel, Olaf

Merlin, Gérard

Merry, Troy L.

Messaritakis, Ippokratis

Mettler, Liselotte

Metz, Luanne

Metzger, Dennis W.

Metzinger, Laurent

Metzler, Markus

Metzler-Wilson, Kristen

Meulenberg, Eline

Meuris, Bart

Meyer, Barbara J.

Meyers, Gregor

Mian, M.A. Rouf

Miccadei, Stefania

Michalek, Katarzyna

Michela, Castagna

Michelhaugh, Sharon K.

Michels, Aaron

Michetti, Fabrizio

Michiels, Carine

Michl, Thomas D.

Michos, A.

Mico, Juan Antonio
Middelburg, T.A.

Middeldorp, Christel M.

Midgley, Adam

Miele, Claudia

Migliaccio, Anna Rita

Miguel, Graça

Miguel, Leiva-Brondo

Miki, Yasuhiro

Mikoshiba, Katsuhiko

Milic, Natasa

Milione, Massimo

Millecamps, Magali

Milledge, John J.

Miller, Aaron W.

Miller, Alyson

Miller, John

Milling, Simon

Milner, Caroline M.

Milner, Joel

Mimaki, Yoshihiro

Mimura, Tetsuro

Minagar, Alireza

Minakata, Daisuke

Minami, Ichiro

Minarchick, Valerie

Minbiole, Kevin P.C.

Mindt, Thomas L.

Mine, Yuichi

Minelli, Alba

Mingorance, Jesús

Minor, Philip

Miquel, Barceló-Oliver

Miranda, Carlos

Miron, Richard J.

Mirzayans, Razmik

Misevic, Gradimir N.

Mishra, Nigam

Mishra, Yogendra Kumar

Mishur, Robert J.

Misra, Biswapriya B.

Misu, Tatsuro

Mitani, Akio

Mitchell, Scott G.

Mitra, Abhisek

Mitra, Raja

Mitsunaga, Shuichi

Mittnacht, Sibylle

Miura, Nobuhiko

Miyagawa, S.

Miyake, Sachiko

Miyake, Shiro

Miyamoto, Koji
Miyamoto, Takeshi

Miyamoto, Toshinobu

Miyamoto, Yohei

Miyata, Jun

Miyata, Yasuyoshi

Miyazaki, Daigo

Miyazaki, Teruo

Miyazaki, Tetsuro

Miyoshi, Daisuke

Miyoshi, Eiji

Miyoshi, Hiroaki

Miyoshi, Norio

Mizoguchi, Toshihide

Mizuo, Keisuke

Mo, Huanbiao

Moal, Iain

Mobed-Miremadi, Maryam

Mocellin, Simone

Modirrousta, Mandana

Moeller, Hanne B.

Moffet, David

Mogi, Masaki

Moham, Aza

Mohamed, Riyaz

Mohammed, Sulma

Mohanam, Sanjeeva

Mohanram, $\mathrm{H}$.

Mohanta, Tapan

Mohanty, Smruti R.

Möhlendick, Birte

Mohn, Dirk

Molasiotis, Athanasios

Moldzio, Rudolf

Möller, Heiko

Molnar, Ferdinand

Molyneux, Karen

Monaco, Edward A., III

Moncalián, Gabriel

Monchau, Francine

Monedero, Vicente

Moniche, F.

Monji, Akira

Monk, Jennifer

Monsalve, Maria

Montagné, Nicolas

Monteil-Rivera, Fanny

Montenegro, Lucia

Montenegro, Maria F

Montes Resano, Marta

Montoya, Guillermo

Moon, Andrea F.

Moon, Il Soo 
Moore, Robert J.

Moos, Philip

Morales, Juan C.

Morales, Julio

Morales-Cruz, Abraham

Morales-Santana, Sonia

Morán, Paloma

Moran, Yehu

Morandi, Luca

Morbidelli, Lucia

Morbidelli, Massimo

Moreau, Regis

Moreira, António S.

Moreira, Maria Teresa

Morel, Jean-Benoit

Morello, Roy

Morgan, Iain

Morgat, Clément

Morgutti, Silvia

Mori, Kiyoshi

Mori, Masaaki

Mori, Matteo

Mori, Seiichiro

Mori, Yoshiharu

Moriguchi, Takaya

Morikawa, Toshio

Morita, Kyoji

Morley, Steven Douglas

Moro, Loredana

Morreau, Hans

Morrill, Jason A.

Morris, Brian J.

Morrow, Jennifer

Morshead, Cindi M.

Mortimer, Peter

Moschetta, Michele

Moschovi, Maria

Moseke, Claus

Mosher, M.J.

Mothe, Beatriz

Motorine, Iouri

Moura, Andre

Mouradian, M. Maral

Mouradov, Aidyn

Mousa, Shaaban A.

Moustris, Kostas

$\mathrm{Mu}$, Dezhi

Muccilli, Vera

Mühl, Heiko

Muinelo, Laura

Muir, Keith

Muise-Helmericks, Robin C.
Mujagic, Zlatan

Mukaida, Naofumi

Mukherjea, Debashree

Mukherjee, Somnath

Müller, Caroline

Müller, Christian P.

Müller, Dafne

Muller, Patricia A.J.

Mullin, James M.

Munang'andu, Hetron M.

Mungamuri, Sathish-Kumar

Munoz-Amatriain, Maria

Murakami, Yasufumi

Murakami, Yoshiki

Muralidharan-Chari, V.

Murashita, Koji

Murata, Yoshiyuki

Murillo, Ana Gabriela

Murono, Shigeyuki

Murphy, Andrew

Murphy, Michael E.P.

Murphy, Paula

Murray Stewart, Tracy

Murray, S.A.

Murray, Thomas Scot

Murugaiyan, Jayaseelan

Musch, Mark

Musio, Carlo

Mustafa, Ghazala

Mustafa, Sanam

Musumeci, Giuseppe

Muthana, Munitta

Mutoh, Michihiro

Mutsaers, Steven E.

Muylaert, Dimitri E.P.

Muylkens, Benoît

Myers, Damian E.

Myers, Daniel D.

Myers, Linda K.

Mysliwiec, Tami

Nachappa, Punya

Nadiminty, Nagalakshmi

Nadler, Jerry L.

Naegele, R.P.

Naftalin, Richard

Nagai, Kouhei

Nagai, Takayuki

Nagai, Takeshi

Nagai, Yoshinori

Nagaraju, Ganji P.

Nagatoishi, Satoru

Nagel, Raimund
Nagelhus, Erlend Arnulf

Nagpal, Seema

Nagwekar, Janhavi

Nagy, Laszlo

Nah, Seung-Yeol

Nahashon, Samuel

Najimi, Mustapha

Naka, Hiroaki

Nakagawa, Shunsaku

Nakagawa, Yoshimi

Nakahama, Ken-ichi

Nakahara, Kazuhiko

Nakajima, Hiroko

Nakaki, Toshio

Nakamoto, Masatoshi

Nakamoto, Shingo

Nakamura, Kazufumi

Nakamura, Masato

Nakamura, Noriko

Nakamura, Shingo

Nakamura, Takahiro

Nakamura, Tsukasa

Nakamura, Yoji

Nakano, Kazuhiko

Nakano, Miyako

Nakashima, Kazuo

Nakashima, Souichi

Nakashiro, Koh-ichi

Nakatani, Yoshihiko

Nakatsuka, Takashi

Nakayama, Takayuki

Nakayama, Yuji

Nakazawa, Masami

Nalam, Vamsi

Nam, Kyoung Hee

Nanda, Himansu

Nandi, Saikat

Nandwana, Vikas

Nanjappa, Manjunatha

Nanni, Loris

Naqvi, Afsar

Narani, Akash

Narayana, Ashwatha

Narayanan, Anand

Narayanasamy, Prabagaran

Nardelli, Dean T.

Nardon, Chiara

Narendhirakannan, R.T.

Narod, Steven A.

Narute, Purushottam

Nascimbeni, Fabio

Nascimento, Alessandro S. 
Nasopoulou, Constantina

Nasrallah, June B.

Nass, Norbert

Nassuth, Annette

Nastro, Rosa Anna

Natalello, Antonino

Natarajan, Sathish Kumar

Natesan, Senthil

Nathanson, S. David

Navarra, Michele

Navarro, Esther Pérez

Naviglio, Silvio

Naya, Francisco

Nazar, Ross N.

Nazaruk, Jolanta

Nazzal, Sami

Nde, Divine B.

Nedachi, Taku

Negre-Salvayre, Anne

Nelson, Fred R.T.

Nemenoff, Raphael

Nenoi, Mitsuru

Neri, Simona

Nesmelov, Yuri E.

Neumann, Martin

Neureiter, Daniel

Newman, David

$\mathrm{Ng}$, Chau Hsien Matthew

$\mathrm{Ng}$, Hui Wen

NG, Kevin Tak-Pan

$\mathrm{Ng}$, Say Kong

Nguyen, Dan-Vinh

Nguyen, Linh Thuy

Nguyen, Phuong

Nguyen, Ruby H.N.

Nguyen, Thomas T.

$\mathrm{Ni}$, Weiming

Nianiou-Obeidat, Irini

Nibbs, Robert J.B.

Nicholson, John W.

Nicholson, Louise F.B.

Nickerson, Scott

Nicolaides, Theodore

Nicolás, Francisco E.

Nicoletti, Ferdinando

Nicoloso, Milena S.

Nie, Xianzhou

Niederman, Robert A.

Nierkens, Stefan

Nieto, Gema

Nifli, Artemissia-Phoebe

Nigatu, Yeshambel T.
Nigris, Filomena De

Niidome, Takuro

Niikura, Masahiro

Nijnik, Anastasia

Nikamo, Pernilla

Nilsson, Gunnar

Ning, Ke

Ninomiya, Kiyofumi

Nishida, Naoki

Nishiguchi, Masamichi

Nishimura, Kaneyasu

Nishimura, Noriyuki

Nishimura, Taisuke

Nishina, Hiroshi

Nishitani, Chikako

Nishiwaki, Hisashi

Nishiyama, Akiko

Nissen, Peter H.

Nistala, Ravi

Nisticò, Roberto

Nitulescu, George Mihai

Niwa, Rieko

Niyibizi, Christopher

Njoku, Dolores B.

Nocentini, Giuseppe

Nociari, Marcelo

Noël, Emily S.

Nofer, Jerzy-Roch

Noghero, Alessio

Noguchi, Constance Tom

Noguchi, Ko

Noh, Ji Heon

Nojiri, Takashi

Nomura, M.

Nomura, Takeo

Nonami, Atsushi

Nonappa, Nonappa

Nonnemacher, Michael R.

Norman, Trevor

Noseda, Diego Gabriel

Novack, Gary D.

Novák, Jan

Novak, Richard

Nowak, Michael

Noyes, Richard D.

Nozell, Susan E.

Nozik-Grayck, Eva

Ntie-Kang, Fidele

Nuevo, Michel

Nunes, Cláudia

Nunomura, Wataru

Nuthikattu, Saivageethi
Nylander, Karin

O'Brien, Kristen

Obata, Yasuko

Obermair, Gerald J.

Obermüller, Nicholas

O'Brien, Nora

Occelli, Florent

Occhialini, Alessandro

Ochiai, Tsuyoshi

Ochiya, Takahiro

O'Connor, James

O'Connor, John J.

O'Connor, Kathleen L.

O'Connor, Timothy P.

Odriozola-Serrano, Isabel

Oertel, Michael

Oertel, R.

Ogasawara, Toru

Ogata, Tadanori

Ogawa, Hidehiko

Ogbourne, Steven M.

Ogi, Kazuhiro

Ogino, Shuji

Ogris, Manfred

Oh, Daniel S.

Oh, Dong-Chan

Oh, Joo Youn

Oh, Jun

Oh, Keimei

Oh, Sangtaek

Oh, Yohan

Oh, Yoon Sin

O'Hara, Steven

Ohe, Kenji

Ohm, Jae-Bom

Ohmiya, Akemi

Ohnishi, Masatoshi

Ohno, Sho

Ohri, Sujata Saraswat

Ohta, Yoshiji

Oikawa, Akira

Oji, Yusuke

Okada, Tomoyo

Okada, Yukinori

Okayasu, Ryuichi

Okegawa, Takatsugu

Okello, Edward

Oklu, Rahmi

Oksanen, Esko

Oku, Hiromi

Okumura, Kazuhiko

Okumura, Tadayoshi 
Olabisi, Opeyemi

Olajide, Olumayokun

O'Leary, Stephen

Oleinick, Nancy

Olivares-Navarrete, René

Olivecrona, Gunilla

Oliveira, Gustavo M.S.

Oliveira, Joana

Oliveira, P.A.

Oliveira-Ferrer, Leticia

Oliver, Brian G.

Oliver, Francisco Javier

Oliver, Richard

Oliver, Stefan

Olivier, Micheau

Olmedillas López, Susana

Olsen, Nancy

Olson, Michael E.

Olson, Mira S.

Oltean, Sebastian

Oltra, Elisa

Omar, Syed Haris

Omi, Toshinori

Omoruyi, Felix

Ono, Akira

Onyenwoke, Rob U.

Oo, Ye Htun

Ooi, Jenny

Oon, Hazel H.

Opdenakker, Ghislain

Opitz, Jörg

Oppenheimer, Steven B.

Orban, Laszlo

Orciani, Monia

Ordónez-Mena, José M.

Oresnik, Ivan J.

Orlandi, Augusto

Orlando, Giuseppe

Orlova, Elena V.

Orsi, Andrea

Ortega, Angel L.

Orth, James D.

Orucevic, Amila

Osakada, Kohtaro

Osaki, Mitsuhiko

Oshitari, Toshiyuki

Oskeritzian, Carole A.

Oster, Henrik

Otagaki, Shungo

Othman, Ahmad Sofiman

Otsuka, Fuminori

Ottewell, Penny
Ottmann, Christian

Otto, Angela M.

Oursler, Merry Jo

Ovilo, Cristina

Owen, Carolyn

Oyoshi, Takanori

Ozaki, Iwata

Ozawa, Yoko

Ozeki, Nobuaki

Ozeki, Yoshihiro

Ozen, Mustafa

Ozturk, Can

Pacak, Andrzej

Pace, Betty S.

Paci, Maurizio

Pacios, Luis

Padmanabhan, Jaya

Padrela, Luis

Padula, Matthew

Pagano, Luca

Pai, Balagopal

Paik, Hyun-Dong

Pajak, Paulina

Palacios, Daniela

Palacios, José-Manuel

Palazzo, Elisabetta

Palenzuela López, J.A.

Palese, Peter

Palitti, Fabrizio

Palmieri, Dario

Palmirotta, Raffaele

Palomares, Eva Rufino

Palumbo, Carla

Pan, Hung-Chuan

Panabieres, Franck

Panáková, Daniela

Panara, Francesco

Panaro, Maria Antonietta

Panchapakesan, Balaji

Panda, Amaresh C.

Pandolfi, Assunta

Panee, Jun

Paneni, Francesco

Panfoli, Isabella

Pang, Myung-Geol

Pani, Bibhusita

Panicker, Rajesh

Panico, Antonio

Panizzutti, Bruna

Panse, Rozen Le

Panzella, Lucia

Pao, Ching-I
Paolocci, Nazareno

Papaccio, Gianpaolo

Papadakis, Raffaello

Papadimitriou, Vassiliki

Papadimitriuou, K.

Papadopoulos, Petros

Papenbrock, Jutta

Papi, Alessio

Papke, Roger L.

Paquete, Catarina M.

Paragas, Neal

Parahuleva, Mariana S.

Parakhonskiy, B.V.

Parakhonskiy, Bogdan

Pardo, María

Paredes, Estefanía

Parinandi, Narasimham L.

Park, Hyun-Woo

Park, Jiyang

Park, Jong-Hoon

Park, Jung

Park, Ky Young

Park, Seong Yong

Park, Taehwan

Park, YongKeun

Park, Yong-Koo

Parker Lemieux, KiTani

Parker, Caroline

Parker, Dane

Parkes, Maria

Parks, William

Parletta, Natalie

Parola, Maurizio

Partanen, J.

Partonen, Timo

Pasini, Luigi

Paslakis, Georgios

Passarella, Daniele

Passi, Alberto

Pastor, Fernando

Pastores, Gregory M.

Pastori, Ricardo L.

Patel, Daxesh P.

Patel, Keyur

Patel, Niketa

Patel, Yashomati M.

Pathak, Harsh

Patil, Sunil A.

Pattanaik, Sitakanta

Patterson, Andrew

Paul, Thornalley

Paulmurugan, Ramasamy 
Pauly, Nicolas

Paz-Filho, Gilberto

Pazzagli, Luigia

Pearen, Michael

Pechanova, Olga

Pecinka, Ales

Pedersen, Anders

Pedersen, Lea

Pedersen, Mangor

Pedeux, Rémy

Pedroza, Mesias

Peijnenburg, Willie

Peinado, Juan R.

Peitzsch, Claudia

Pelagalli, Alessandra

Pellei, Maura

Pelletier, Stephane

Pelosi, Paolo

Penfornis, Patrice

Peng, Chien-Fang

Peng, Robert

Peng, Sheng-Bin

Peng, Wen-Huang

Pennypacker, Keith

Pentzold, Stefan

Peppelenbosch, Maikel

Perán, Macarena

Perazzolli, Michele

Percival, Susan

Pereira, C.

Pereira, David

Pereira, Olívia R.

Pereira, Susana

Perepelyuk, Maryna

Perera, Omaththage P.

Perez-Cano, Francisco

Pérez-Castrillón, José L.

Pérez-Correa, José Ricardo

Pérez-Jiménez, A.J.

Perez-Jimenez, Jara

Pérez-Montaño, Francisco

Perez-Stable, Carlos

Péros, Jean-Pierre

Perrais, Michaël

Perreault, Jonathan

Perricone, Carlo

Perrier, Josette

Perry, Mark M.

Persson, Rasmus

Pesis, Edna

Petersen, Björn

Peterson, Daniel
Peterson, Eliza J.R.

Peterson, Steve

Petitot, Anne-Sophie

Petropoulos, Isabelle

Petrosyan, Armen

Pettersen, Caroline

Pettit, Allison

Peulen, Olivier

Pezzella, Alessandro

Pfeffer, Larry

Phanstiel, Otto

Phatarpekar, Prasad

Phelps, Patricia E.

Philips, Neena

Phillips, Jacqueline K.

Phillips, James B.

Phillips, Robert $S$.

Phillips-Jones, Mary K.

Phipps, Richard P.

Pi, Liya

Piazza, Fabrizio

Picariello, Gianluca

Piccialli, Gennaro

Pichler, Martin

Pickett, Stephen D.

Pickova, Jana

Picorel Castaño, Rafael

Pidko, Evgeny A.

Pierini, Antonio

Piggins, Hugh D.

Pignata, Claudio

Pignataro, Giuseppe

Pignolo, Robert J.

Piiper, Albrecht

Pikart, Tiago Georg

Pilati, Stefania

Pilishvili, Tamara

Pilkington, Suzanne M.

Pillay, Viness

Pilon-Thomas, Shari

Pineault, Nicolas

Pineda, Miguel

Pinto, Antonio

Pinzani, Pamela

Piomboni, Paola

Pioszak, Augen

Piróg, Katarzyna A.

Pirola, Luciano

Pisabarro, Antonio G.

Pisani, Antonio

Pisitkun, Trairak

Pislariu, Catalina
Pisoni, Giorgia Brambilla

Pitto, Letizia

Pitto-Barry, Anaïs

Piva, Terrence

Pivetal, Jérémy

Pixley, Sarah

Pizzo, Elio

Plantinga, Theo

Platt, Jeffrey L.

Platts, James

Plaza, Gustavo R.

Plazzi, Federico

Pliego-Alfaro, Fernando

Plock, Jan A.

Ploughman, Michelle

Pochynyuk, Oleh

Poddar, Ranjana

Podszun, Maren

Poelstra, Jelmer

Poinsot, Verena

Poirier, Guy G.

Poirier, Raymond A.

Poirot, Marc

Poldermans, Don

Poleszak, Ewa

Polette, Myriam

Poli, Alessandro

Poli, Guido

Pollitt, Krystal

Polticelli, Fabio

Polyak, Stephen J.

Pommer, Bernhard

Ponnambalam, Sreenivasan

Pons, Antoni

Pons, Tirso

Popa-Wagner, Aurel

Popescu, Nirvana

Porpora, Maria Grazia

Porteu, Françoise

Posé, David

Post, Janine

Potterat, Olivier

Pouliot, Normand

Poulsen, Anders

Pountney, Dean

Pourahmad, Jalal

Pourquier, Philippe

Powell, Daniel

Powers, Evan T.

Pozo, Óscar J.

Pozzobon, Michela

Prante, Olaf 
Prats, Anne-Catherine

Pratsinis, Harris

Prchal, Josef

Preckel, Benedikt

Preissner, Klaus T.

Prentice, Howard

Proctor, Richard A.

Proestos, Charalampos

Proudfoot, Amanda E.I.

Provost, Patrick

Pruszyński, Marek

Prykhodko, Olena

$\mathrm{Pu}$, Christy

$\mathrm{Pu}$, Jing

Pucci, Ida

Puddu, Paolo Emilio

Puglia, Debora

Puglisi, Fabio

Pühler, Alfred

Puiggalí, J.

Puiggalí, Jordi

Pulati, Nuerxida

Pulido, David

Pulignani, Silvia

Pulinilkunnil, Thomas

Pulliainen, Arto

Pumera, Martin

Puri, Akshit

Putz, Mihai V.

Puvvula, Pavan Kumar

Qaderi, Mirwais M.

Qi, Robert Z.

Qi, Xin

Qian, Li

Qian, Pengxu

Qian, Wei

Qin, Xue-Mei

Qiu, Huan

Qiu, Jianwen

Qiu, Wei

Quail, Daniela F.

Quan, Guoxing

Quan, Taihao

Quesada, Víctor

Quigley, Eamon

Quigley, Eamonn

Quillard, Thibaut

Quinn, Julian

Quintana, José

Quintana, M.C.

Rachek, Lyudmila

Rachmawati, Dessy
Racioppi, Luigi

Rad, Behzad

Radhakrishnan, Ravi

Rådinger, Madeleine

Radner, Franz

Radons, Jurgen

Radwan, Osman E.

Raff, Johannes

Raffaelli, Marco

Raghupathi, Wullianallur

Ragsdale, David

Ragusa, Maria Antonietta

Rahimi, Farid

Rahman, Ziaur

Rai, Padmalatha S.

Raimondi, Lavinia

Rajaputra, Pallavi

Rajarathnam, Krishna

Rajasekaran, Parthiban

Rajkkumar, Premraj

Rajput, Ashwani

Rakofsky, Jeffrey J.

Rakotondrafara, Aurelie

Rakus, Dariusz

Rama, Paolo

Ramachandran, Srinivasan

Ramadan, Qasem

Ramakrishna, Suresh

Ramalingam, Latha

Raman, Sripriya

Ramani, Komal

Ramani, N.V.

Ramaraj, Pandurangan

Ramidi, Punnamchandar

Ramírez, José M.

Ramis, Rebeca

Ramón, Daniel

Ramonda, Roberta

Ramos, Sonia

Ramos-Morales, Francisco

Ramos-Vivas, José

Ramzi, Mohammed

Ran, Chongzhao

Rana, Dipak

Rand, Matthew

Randall, Stephen K.

Ranganathan, Natarajan

Ranieri, Elena

Ranzato, Elia

Rao, C.V.

Rao, Rosa

Raposo, Maria Filomena Jesus
Rappaport, Jay

Rasche, Leo

Raspanti,, Mario

Rassoulzadegan, Minoo

Rastegar, Sepand

Ratcliffe, R. George

Raterman, H G Raterman

Raucher, Drazen

Ravegnini, Gloria

Ravid, Katya

Rawashdeh, Oliver

Rawel, Harshadrai

Rawls, Henry R.

Ray, Partha

Ray, Ranjit

Raynes, J.K.

Razumilava, Nataliya

Rea, Katia

Reading, Benjamin J.

Reale, Marcella

Rebelo, Irene

Rebl, Alexander

Rebollar, Esther

Redden, Robert

Reddy, Narsa M.

Reddy, Sakamuri

Reddy, Venkateshwar

Redell, Michele

Redon, Christophe E.

Reeve, Amy

Reeve, Vivienne

Regenbrecht, Christian R.A.

Rehrig, Erin M.

Reid, Dugald

Reijns, Martin

Reinach, Peter S.

Reinders, Joerg

Reindl, Markus

Reineke, Annette

Reinhardt, Dirk

Reisman, Scott A.

Reissmann, Siegmund

Remuzzi, Giuseppe

Ren, Jianguo

Ren, Natalie

Ren, Shuxin

Renaudineau, Yves

Rendeiro, Catarina

Renner, Ulrich

Rennert, C.

Renshaw, Stephen A.

Renzoni, Elizabeth 
Reshkin, Stephan Joel

Revelli, Luca

Revilla, Eugenio

Rex, Tonia

Rey, A.I.

Rey, Patrice

Rezzani, Rita

Rhazi, Larbi

Rhee, Ki-Jong

Rialland, Mickaël

Riazuddin, S.

Ribeiro, Isabel

Ribeiro, Rogerio

Ribeiro, Susana

Ribeiro-Barros, Ana I.

Ricciardelli, Carmela

Rich, Alison

Richards, Renée Stirling

Richardson, Alan

Richardson, Mark F.

Rider, Cynthia

Rieder, Michael

Riedl, Rainer

Rief, Harald

Rieg, Timo M.

Rieger-Christ, Kimberly M.

Riess, Olaf

Rigas, Stamatis

Rimondini, Lia

Rimstad, Espen

Rinaldo, Charles R.

Rink, Cameron

Rinnerthaler, Gabriel

Riou, Laurent M.

Risk, Janet M.

Rittner, Heike

Ritze, Yvonne

Ro, Simon Weonsang

Roan, Nadia R.

Roato, Ilaria

Roberto, Vânia

Roberts, Ashley

Robey, Pamela

Robinson, Brooks

Robledo-Sierra, Jairo

Robson, Kathryn

Roccheri, Maria Carmela

Rocha, Sonia

Rocha-Ferreira, E.

Roche, Daniel

Rödel, Franz

Rodicio, María Celina
Rodino-Klapac, Louise R.

Rodnina, Marina

Rodrigo, Ismael

Rodrigues, Thaís Barros

Rodríguez, Amaia

Rodriguez, Cristina P.

Rodríguez, Manuel José

Rodríguez-Couto, Susana

Rodríguez-Pascual, Fernando

Rodriguez-Revenga, Laia

Roehe, Rainer

Roelen, Bernard

Roesch, Frank

Roessner, Ute

Roffler, Steve R.

Rogers, Hilary

Rogister, Bernard

Roh, Changhyun

Roh, Sangho

Rohira, Aarti

Romalde, Jesus

Romano, Federica

Romanov, Victor

Romero Martínez, Ángel

Romero, Francisco Javier

Romero, Maria-Paz

Rommer, Paulus

Rompel, Annette

Roncada, Paola

Rondanino, Christine

Rong, Yueguang

Roos, Jessica

Roque, Cláudio

Ros Lis, Jose Vicente

Rosado, Juan

Rosario, Karyna

Rose, Aaron $\mathrm{H}$.

Rose, Alan B.

Rose, Christopher F.

Roseiro, Luísa B.

Rosell, Rafael

Rosellini, Daniele

Roselló, Salvador

Rosenberg, Helene

Rosendahl, Jonas

Rosenmann, Hanna

Rosenthal, Philip

Rosenwasser, Alan M.

Rosenzweig, Derek

Rosi, Antonella

Ross, Sharon

Rossello, Armando
Rossi, Simona

Rossignol, Julien

Rostaing, Lionel

Rouhana, Labib

Routier, Françoise H.

Rovaris, Marco

Rovida, Elisabetta

Rowland, Ian

Rowland, Lisa

Roy, Jérôme

Roy, Nilotpal

Roy, Peter J.

Roy, Santanu

Roy, Somak

Roy, Sourav

Roychowdhury, Sanjoy

Rozhkova, Elena

Rozhon, Wilfried

Ruan, Xiangbo

Rubakhin, Stanislav

Rubert, Josep Rubert

Rudkin, Brian

Rudyk, Olena

Ruff, Adrian

Ruggeri, Paolo

Ruggerone, Paolo

Ruiu, Luca

Ruiz, José

Ruiz, Oscar A.

Ruiz, T.

Ruiz-Echevarria, Maria J.

Rund, Deborah

Runte, Christoph

Ruppert, J. Michael

Rupprecht, Rainer

Ruseva, Marieta

Rush, Demaretta S.

Rusnati, Marco

Ruso, Juan

Russ, Brendan

Russell, Fraser D.

Russell, Joanne

Russo, Antonio

Russo, Maria

Russo, Matteo A.

Russu, Wade A.

Rutgers, Michiel

Rüther, Thomas

Ruthstein, Sharon

Rutkowski, Joseph

Ryan, David K.

Rybak, Leonard 
Rychkov, Grigori

Ryckman, Kelli K.

Ryoo, Hyun-Mo

Sá, Rosália

Saba, Nakhle S.

Sacchetti, Lucia

Saccone, Valentina

Sachse, Frank B.

Sadakane, Masahiro

Sadik, Riadh

Sadkowski, Tomasz

Sadovsky, Yoel

Saeki, Ikuyo

Saez, Carmen

Safa, Ahmad

Saferding, Victoria

Sagi, Amir

Saher, Gesine

Sahin, Özgür

Sahlén, Pelin

Sahrawy, Mariam

Sahrmann, Philipp

Sahu, Saura C.

Saiano, Filippo

Saikumar, Pothana

Sainaghi, Pier Paolo

Saini, Sharanjot

Sainlos, Matthieu

Saisho, Yoshifumi

Saito, Hidetsugu

Saito, Hiroshi

Saito, Yoshinori

Saito, Yoshiro

Saitoh, Akiyoshi

Saitoh, Masao

Sajeva, Maurizio

Sajic, Marija

Sakai, Atsushi

Sakakura, Akira

Sakuma, Kunihiro

Sakurai, Manabu

Sakwe, Amos

Salas-de La Cruz, David

Salazar, Gloria

Saldaña, Guillermo

Salek, Reza

Salgado, Josefa

Salhia, Bodour

Salih, Erdjan

Salim, Vonny

Salini, Michael J.

Salinthone, S.
Sal-Man, Neta

Salomone, Federico

Saltel, Frédéric

Salton, Maayan

Saluja, Ashok

Salvati, Bruno

Salvato, Fernanda

Salvatore, Ralph Nicholas

Salvatore, Silvia

Samalin, E.

Samant, Tanay S.

Samarakoon, R.

Samuel, Temesgen

Samuels, Mark E.

San Martin, Carmen

Sánchez Alcázar, José

Sanchez, David V.P.

Sanchez, Diego H.

Sanchez, Goar

Sánchez, Sandra

Sanchez-Dalmau, Bernardo F.

Sanchez-Garcia, Isidro

Sánchez-Moreno, Manuel

Sancho, Rocio

Sangelaji, Bahram

Sanjust, Enrico

Sanna, Fabio

Sano, Shigetoshi

Santamaría, Estrella

Santarelli, Lory

Santella, Luigia

Santer, Frédéric R.

Santini, Valeria

Santino, Angelo

Santo, Carmela De

Santoni, Matteo

Santos, Ana Paula

Santos, Tírcia C.

Santos-Gallego, Carlos

Santra, Dipak K.

Santulli, Gaetano

Sarath, Gautam

Sarkar, Chinmoy

Sarker, Dipak

Sarnyai, Z.

Sasaki, Masanori

Sasaki, Sei

Sasazuki, Shizuka

Sato, Masahiro

Sato, Minoru

Sato, Trey K.

Sato, Tsutomu
Sato, Yuichiro

Satoh, Shigeru

Sato-Nara, Kumi

Sator-Katzenschlager, Sabine

Satou, Ryousuke

Sattar, Sampurna

Sattler, Rita

Saura, María

Saux, Olivier

Savage, David B.

Savaraj, Niramol

Saville, Robert

Savoia, Dianella

Savopoulos, Christos

Sawada, Hiroshi

Sawada, Kenjiro

Sawai, Hirofumi

Saxena, Praveen

Sayers, Thomas J.

Scafoglio, Claudio

Scalise, Mariafrancesca

Scanes, Colin G.

Scarlata, Suzanne

Schachter, Michael

Schaefer, Jeremy

Schaefer, Michael

Schaffler, Mitchell B.

Schäffner, Anton

Schalken, Jack

Schatten, Heide

Schausberger, Peter

Scheffler, Tracy L.

Scheiner, Stefan

Scheinman, Robert

Scheper, Verena

Scherer, Günther

Schiefer, Isaac T.

Schierwater, Bernd

Schiffmann, Raphael

Schindler, Thomas

Schinke, Thorsten

Schirmer, Bastian

Schjoldager, Katrine T.-B.G.

Schlaepfer, Isabel R.

Schmid, Markus

Schmidt, Jochen

Schmidt, Monica

Schmidt-Arras, Dirk

Schmitt, Joachim

Schmittgen, Tom

Schmitz, Gerd

Schmitz-Linneweber, C. 
Schnackenberg, Laura

Schneider, Barbara

Schneider, Bernd

Schneider, David

Schneider, Jochen

Schneider, Raphaël

Schoenhagen, Paul

Scholz, Glen

Scholz, Sonja

Schonthal, Axel

Schoonheim, Menno M.

Schrag, Matthew

Schraufstatter, Ingrid U.

Schrauwen-Hinderling, V.B.

Schrempf, Alexandra

Schrittwieser, Stefan

Schroeder, Dana

Schroeder, Grzegorz

Schroeder, Hilke

Schroeter, Michael

Schulenburg, Hinrich

Schulz, Benjamin L.

Schulz, Wolfgang A.

Schwartz, Gary

Schwarz, Steven M.

Schweiger, Michal R.

Schwiebs, Anja

Sciarretta, Sebastiano

Scicchitano, Pietro

Scorziello, Antonella

Scott, Daniel

Scott, Naomi

Scotti, Luciana

Scovassi, Anna Ivana

Scozzafava, Andrea

Scudiero, Rosaria

Sebastiani, Federico

Seca, Ana

Seca, Ana M.L.

Sedger, Lisa

Seegmiller, Adam C.

Seela, Frank

Seeliger, Claudine

Seeling, Joni M.

Seeman, Mary V.

Sefat, Farshid

Sehorn, Michael

Sehrawat, Archana

Seib, Philipp

Seifalian, Alexander M.

Seiliez, Iban

Seiquer, Isabel
Seki, Naohiko

Sekine, KenTaro

Sekino, Masashi

Selim, Sameh

Sella, Luca

Sellam, Adnane

Sellayah, Dyan

Sellner, Johann

Seltsam, Axel

Selvamani, Amutha

Sempere, Lorenzo

Sen, Nilkantha

Sen, Stephanie E.

Sena, Dayse F.

Senga, Takeshi

Senneville, Éric

Senpuku, Hidenobu

Seo, Hak Soo

Serafini, Gianluca

Serafini, Nicolas

Sergeant, Kjell

Seroussi, Eyal

Serra, Francisca

Serrano, Maria

Servillo, Giuseppe

Sestili, Sara

Sethi, Gautam

Setyawati, Magdiel Inggrid

Seva, Catherine

Sgadò, Paola

Sha, Zhe

Shafirstein, Gal

Shah, Dilip

Shah, Nilay

Shaikh, Mohd. Farooq

Shaio, Young-Ji

Shankar, Neelaabh

Shanmugam, Muruganandan

Shao, Kan

Sharma, Amrish

Sharma, Atul

Sharma, Bhesh

Shaul, Yoav D.

Shavrukov, Yuri

Shear, Neil H.

Sheikh, Nadeem

Shemanko, Carrie

Shemfe, Mobolaji

Shen, Haihong

Shen, Leslie

Shen, Tang-Long

Shen, Yang
Shenderovich, Ilya G.

Sherbet, G.V.

Sherer, Nathan

Sheridan, Paul

Sherman, Michael P.

Sherrard, Rachel

Shiau, Chyuan-Yuan

Shieh, Tzong-Ming

Shieh, Wann-Yun

Shikata, Masahito

Shil, Suranjan

Shim, Jung Ok

Shim, Yhong-Hee

Shimizu, Ippei

Shimizu, Shigeomi

Shimizu, Toshiyuki

Shimobayashi, Mitsugu

Shimoda, Hiroshi

Shimosawa, Tatsuo

Shin, Chan Young

Shin, Jae-Ho

Shin, Kwang-Soo

Shinohara, Russell Takeshi

Shintani, Yasushi

Shiota, Masakazu

Shiozawa, Yusuke

Shipley, Janet

Shiraishi, Takehiko

Shirakami, Yohei

Shirasawa, Kenta

Shirure, Venktesh

Shityakov, Sergey

Shivanna, Binoy

Shivapurkar, Narayan

Shoenfeld, Yehuda

Shoham, Menachem

Shoji, Kensuke

Sholkamy, Eman

Shopsowitz, Kevin

Shoshi, Kikuchia

Shrestha, Lok Kumar

Shrivastava, Abhishek

Shu, Mi Chung

Shumilov, Anatoliy

Sicher, Richard C.

Siddiquee, Tasneem A.

Siddiqui, Rafat A.

Sidi, Yechezkel

Sidorenko, Viktoriya S.

Sidransky, Ellen

Siebel, Christian W.

Sieber, Fritz 
Sieber, Jonas

Siebuhr, A.S.

Siegfried, Kellee

Sieiro, Carmen

Sier, Cornelis

Siewert, Bianka

Sigala, Sandra

Sikkema-Raddatz, Birgit

Silbert, Benjamin

Silikas, Nick

Sill, Heinz

Sillence, Dan

Silljé, Herman H.W.

Silva, Jorge Carvalho

Silva, Luís R.

Silva, Sn

Silverberg, Donald S.

Simecka, Jerry W.

Simeon, Vittorio

Simeone, Kristina

Simirgiotis, Mario

Simon, Scott I.

Simons, Sinno H.P.

Simons-Burnett, Andrean

Simpson, Craig

Simpson, John

Simpson, M.R.

Sims, Ian M.

Singh, Anil

Singh, Ashutosh

Singh, Atul Kumar

Singh, Chandra K.

Singh, Jagdish

Singh, Karan

Singh, Kashmir

Singh, Pramod K.

Singh, Prashant

Singh, Rajan

Singh, Reetu R.

Singh, Seema

Singh, Suveer

Sinha, Amit

Sinnett, Daniel

Sipes, Nisha

Sips, Patrick Y.

Siracusa, Laura

Siristatidis, Charalampos

Sisodia, Sangram S.

Sita, Giulia

Siu, Parco

Siwek, Agata

Škalko-Basnet, Nataša
Skendros, Panagiotis

Skinner, Jonathan

Skot, Leif

Skuli, Nicolas

Skundric, Dusanka S.

Skwarczynski, Mariusz

Sleutel, Mike

Sleutels, Tom

Słoczyńska, Karolina

Slominski, Andrzej

Slovin, Janet $P$.

Slusarenko, Alan J.

Smagula, Stephen F.

Smani, Younes

Smiesko, Martin

Smirnovas, Vytautas

Smit, Jasper V.

Smith, Cody J.

Smith, Darrin

Smith, Kylie J.

Smith, Michael

Smith, Micholas

Smith, Rachel

Smith, Tim A.D.

Smits, Anthal

Smolenski, Grant

Smolock, Christopher J.

Smýkal, Petr

Smyrniotis, Vassilios

Smyth, Lesley A.

Soares, Helena

Sobczak-Thépot, Joëlle

Sobin, Cas

Sobolewski, Marissa

Soggiu, Alessio

Sohl, Christal

Sohn, Kai

Sohrabji, Farida

Sokolova, Viktoriya

Solà Alberich, Rosa

Solano, Francisco

Solecka, Jolanta

Soliman, Khairy M.

Solinís, M. Ángeles

Soltani, Ali

Somasundaram, R.

Somers, David

Sommerer, Nicolas

Son, Chang-Gue

Son, Deok-Soo

Son, Mi-Won

Song, Guoqing
Song, Jia L.

Song, Young Chae

Song, Yuyu

Sonkoly, Enikö

Sonnenberg, Anton S.M.

Sonnweber, Thomas

Sonpavde, Guru

Sonsalla, Patricia K.

Sorce, Silvia

Soreq, Hermona

Soriano, Elena

Sorrentino, Elena

Sosa, Silvio

Sosnowska, Anita

Sotome, Shinichi

Sottnik, Joseph

Soucy, Shannon

Souders, Colby A.

Souidi, Maamar

Soulier, Jean

Southam, Andrew D.

Soveral, Graça

Spanakis, Marios

Spandou, Evangelia

Spanò, Stefania

Sparkes, James

Speck, Peter G.

Spee, Bart

Spence, Tara

Spendier, Kathrin

Spickett, Corinne M.

Spingler, Bernhard

Spiotto, Michael T.

Spizzo, Gilbert

Spormann, Alfred M.

Sprenger, Heike

Spruck, Charles

Srebnik, Simcha

Sreedasyam, Avinash

Sridharan, Vijayalakshmi

Srinivas, Keerthi

Srivastava, Akhil

Srivastava, Rakesh

Srivatsan, Eri S.

Srivenugopal, Kalkunte

Staal, Jens

Stanika, Ruslan

Stanley, Jone

Stanta, Giorgio

Stanton, Robert C.

Staple, Alan

Stec, Jozef 
Steen, Ida Helene

Stefanidou, Maria E.

Stefano, Cassanelli

Stefano, Cesco

Stehle, Jörg H.

Steiner, Joseph P.

Steinhilber, Dieter

Stelinski, Lucasz

Stephenson, Sally-Anne

Steup, Martin

Stevanato, Piergiorgio

Steven, Sebastian

Stevens, Richard G.

Steves, Dr. Claire Joanne

Stewart, Anthony

Stifani, Stefano

Stillaert, Filip

Stilli, Donatella

Stine, Keith J.

Stocco, Carlos

Stock, Christian

Stocker, Reto

Stoelzel, Friedrich

Stone, James R.

Stone, Kari

Stope, Matthias B.

Stopsack, Konrad H.

Storer, Nicholas P.

Stork, Christian J.

Strano, Sabrina

Straub, Rainer H.

Strauss, Sarah

Strekalova, Tatyana

Strekowski, Lucjan

Streng, Andrea

Strick, Reiner

Strickland, Dudley K.

Stritzker, Jochen

Strollo, Felice

Stuart, Jeff

Studzinski, George P.

Stuppia, Liborio

Stura, Enrico A.

Styrsky, John D.

$\mathrm{Su}$, Baofeng

$\mathrm{Su}$, Chunming

$\mathrm{Su}, \mathrm{Pi}-\mathrm{Guey}$

$\mathrm{Su}$, Ruey-Chyi

$\mathrm{Su}$, Ting

$\mathrm{Su}$, Wen-Ta

Suades, Joan

Subramaniam, Prasad
Sucharov, Carmen

Sudhir, Putty-Reddy

Sugimura, Haruhiko

Sukumari-Ramesh, Sangeetha

Sulek, Karolina

Sullards, Cameron

Sumitani, Jun-ichi

Sun, Daekyu

Sun, Fen

Sun, Jie

Sun, Ming

Sun, Mingkuan

Sun, Qi

Sun, Wenyue

Sun, Ying

Sun, Yingjie

Sun, Zhifu

Sundar, Isaac Kirubakaran

Sundd, Prithu

Sung, Patrick

Superti, Fabiana

Supuran, Claudiu T.

Surber, Christian

Surguchov, Andrei

Sussan, Thomas

Sussman, Mark A.

Sutherland, Andrew

Sutton, Timothy A.

Suzuki, Hiroetsu

Suzuki, Hiroshi I.

Suzuki, Nobuhiro

Suzuki, Takuya

Suzuki, Yuichiro J.

Svenja, Hardtke

Svensson, Katrin J.

Swadźba-Kwaśny, M.

Swales, Catherine

Swamidass, S. Joshua

Swarnkar, Gaurav

Swarup, Vimal

Sweazea, Karen

Swerdlow, Russell

Swevers, Luc

Switonski, Marek

Sylla, Maite

Sylvester, Paul W.

Sylvie, Monferran

Szasz, Theodora

Szczerba, Wojciech

Szekely, Gyorgy

Szeto, Yim-Tong

Szyszkowicz, Mieczysław
Taboureau, Olivier

Tabuchi, Yoshiaki

Tacer, Klementina Fon

Tada, Hayato

Tafesse, Fikadu Geta

Tagliabue, Elda

Taguchi, Yoshihiro

Taheri, Arash

Tahriri, Mohammadreza

Tai, Helen H.

Taira, Junsei

Tajmir-Riahi, H.A.

Takabe, Kazuaki

Takagi, Kiyoshi

Takahisa, Kanekiyo

Takaki, Akinobu

Takamatsu, Kazuhiko

Takano, Katsura

Takanori, Maruta

Takao, Sonshin

Takatsuji, Hiroshi

Takayama, Yoshiharu

Takayanagi, Toshiyuki

Takemori, Hiroshi

Takenaga, Mitsuko

Takeshita, Fumitaka

Takeshita, Haruo

Takeuchi, Yuko

Takumi, Shigeo

Takyar, Seyedtaghi

Talbert, Joey N.

Talou, Julián Rodriguez

Tam, Wilson W.S.

Tamaki, Hideyuki

Tamalis, Dimitri

Tamaru, Teruya

Tambi, Richa

Tamborindeguy, Cecilia

Tambur, Anat R.

Tamma, Grazia

Tamori, Akihiro

Tamura, Ken-ichi

Tan, Eng-King

Tan, Yuqing

Tanahashi, Hiroshi

Tanaka, Hiroshi

Tanaka, Nobuyuki

Tanaka, Shigeo

Tanaka, Takashi

Tanaka, Tomoaki

Tanaka, Yoshiyuki

Tang, Bor Luen 
Tang, Damu

Tang, Simon Y.

Tang, Xiaojia

Tang, Xinyan

Tanigawa, Tetsuya

Tanonaka, Kouichi

Tansey, John

Tanzi, E.

Taoka, Yousuke

Tapia, Anibal Alejandro

Tappia, Paramjit S.

Tarantino, Giovanni

Taratula, Olena

Tarro, Giulio

Tasca, Giorgio

Tashima, Kimihito

Tashiro, Hirotaka

Tassone, Pierfrancesco

Tastet, Lionel

Tata, Ada Maria

Tatakis, Dimitris N.

Tate, Rothwelle J.

Tatemoto, Yuji

Tatzelt, Jörg

Taube, Joe

Tauchi, Tetsuzo

Tava, Aldo

Tavazzi, Barbara

Tavío, María Del Mar

Tavornpanich, Saraya

Tay, Chor Yong

Tayebati, Seyed Khosrow

Taylor, Anna M.W.

Taylor, Caroline

Taylor, Graham

Taylor, Janice

Taylor, Zachary S.

Taymans, Jean-Marc

Tchapla, Alain

Teeri, Teemu

Teets, Nicholas M.

Tegeder, Irmgard

Tejero, Jesús

Telugu, Bhanu Prakash V.L.

Temeyer, Kevin B.

Temmam, Sarah

Temussi, Piero A.

Tendulkar, Ketki

Teng, Ru-Jeng

Teng, Shaolei

Tennessen, Jacob A.

Terada, Naoki
Teramoto, Hidetoshi

Terashima, Tomoya

Terracciano, Daniela

Terrier, Olivier

Terry, Randall G.

Teschke, Rolf

Testai, Fernando D.

Tetlow, Ian

Tevosian, Sergei G.

Thannhauser, Theodore W.

Theiss, Carsten

Thelin, Eric

Themstrup, Lotte

Thevelein, Johan

Thèvenod, Frank

Thiery, Denis

Thijssen, Victor L.

Thilmony, Roger

Thomas, Michelle

Thomas, T.J.

Thompson, Kim

Thomsen, Hauke

Thomson, Daniel

Thorin-Trescases, Nathalie

Thorlacius-Ussing, Ole

Thounaojam, Menaka

Thünemann, Andreas

Thyagarajan, Bharat

Tian, Li

Tian, Yingfang

Tickner, Jennifer

Tie, Jeanne

Tihkonov, Vladimir

Tijeira, Marta

Tikkanen, Ritva

Tiley, Laurence

Tilley, Michael

Tilton, Ronald G.

Timoshenko, Alexander V.

Tiribelli, Claudio

Tittiger, Claus

Tliba, Omar

To, Kin-Ying

Tocci, Giuliano

Toietta, Gabriele

Toju, Hirokazu

Tokoro, Chiharu

Tokuyama, Shogo

Toldo, Stefano

Tölli, Hanna

Tolosa, Ezequiel

Tomasetti, Carmine
Tomasetti, Marco

Tomassetti, Antonella

Tomaz, Cândida Teixeira

Tomitaka, Asahi

Tomoaki, Sakamoto

Tomoda, Hiroshi

Tomofuji, Takaaki

Tomonaga, Keizo

Tomsic, Jerneja

Tondi, Gianluca

Tong, Hoang V.

Tong, Iris

Tong, Jingou

Tong, Yat-Ching

Topakas, Evangelos

Tormo, José Rubén

Torras, J.

Torras, Joan

Torre, Juan

Torrens, Francisco

Torres, Carlos F.

Torres, Jaume

Torres, M.I.

Torres-Aleman, Ignacio

Torres-Russotto, Diego

Tortiglione, Claudia

Tortora, P.

Torzewski, Michael

Tosoian, Jeffrey J.

Toth, Eric A.

Touyarot, Katia

Trabace, Luigia

Traber, Maret

Trabolsi, Ali

Tralau, Tewes

Tramice, Annabella

Tramontano, Donatella

Tran, Nhan L.

Tranbarger, Timothy

Traweger, Andreas

Treesukosol, Yada

Treves, Susan

Trevino, Jose

Trevisi, Lucia

Trif, Monica

Trincone, Antonio

Tringali, Corrado

Tringali, Cristina

Tripathi, Prateek

Tristram, Stephen

Trivedi, Darshan

Trivellini, Alice 
Trognitz, Friederike

Trollmann, Regina

Trollope, Alexandra

Trosko, James

Trovato, Francesca Maria

Trovato, Guglielmo

Trzciński, Krzysztof

Tsaftaris, Athanasios

Tsai, Fuu-Jen

Tsai, James

Tsai, Keng-Chang

Tsai, Kun-Ling

Tsai, Sientang

Tsai, Tsung-Yu

Tschierlei, Stefanie

Tse, William K.F.

Tsitsilonis, Ourania E.

Tsoi, James

Tsuchiya, Kosuke

Tsuchiya, Yuichi

Tsuji, Masahiro

Tsuji, Petra

Tsuji, Shoji

Tsujimura, Seiya

Tsunoda, Ikuo

Tsurkan, Mikhail V.

Tsutsui, Shigeyuki

Tsuyama, Naohiro

Tuaillon, E.

Tuchscherr, Lorena

Tucker, Steven

Tuckermann, Jan

Tuda, Midori

Tuffaha, Sami H.

Tuffery-Giraud, Sylvie

Tulkens, Paul

Tumanov, Alexei V.

Tuna, Deniz

Tung, Tao-Hsin

Tunuguntla, Hari

Tuomikoski, Pauliina

Turiel, Maurizio

Turk, Benjamin E.

Turner, Helen

Turner, Neil A.

Turner, Renee

Turpin, Sarah

Turunen, Mikko P.

Tusell, Llibertat

Tuttolomondo, Antonino

Tuyaerts, Sandra

Tyakht, Alexander V.
Tzang, Bor-Show

Tzeng, Nian-Sheng

Tziomalos, Konstantinos

Tziotzios, Christos

Tzortzakis, Nikolaos

Uchida, Shizuka

Uchio, Yuji

Ueda, Akira

Uehara, Tomoya

Ueno, Shu-ichi

Ueta, Ikuo

Uhr, Jonathan W.

Ulisse, Salvatore

Ulivi, Paola

Ullmann, G. Matthias

Ulsh, Brant

Umberger, Reba A.

Umemori, Juzoh

Uno, Tomohide

Urata, Hidehito

Urrestarazu, Jorge

Ussar, Siegfried

Usui, Soichiro

Uysal, Utku

Uzawa, Akiyuki

Vaidya, Bhuvaneshwar

Vaidyanathan, Ganesan

Vaiman, Daniel

Vainio, Eeva J.

Vainonen, Julia

Vajro, Pietro

Valdes, Ana M.

Valdez, Gregorio

Valenti, Luca

Valenti, Piera

Valentinuzzi, Fabio

Valentovic, Monica

Valero, Manuel

Valiante, Vito

Valle, Adamo

Valli, Veronica

Vallières, Luc

Valpuesta, Victoriano

Van Aerschot, Arthur

Van Bodegraven, Ad A.

Van Brabant Smith, Anja

Van Coppenolle, Fabien

Van De Sluis, Bart

Van Den Bergh, Hubert

Van Den Meiracker, Anton H.

Van Der Heiden, Kim

Van Der Kamp, Marc W.
Van Der Kuyl, Antoinette

Van Der Schee, Marc Philippe

Van Der Spoel, Aarnoud C.

Van Der Veer, Eric P.

Van Dijk, Bart G.M.

Van Etten, Jamie

Van Hemelrijck, Mieke

Van Leeuwen, J.

Van Leeuwen, Thomas

Van Munster, Jolanda M.

Van Oers, Nicolai

Van Putten, Michel J.A.M.

Van Raaij, Mark J.

Van Rijn, Richard M.

Van Werkhoven, Cornelis H.

Van Westering, Tirsa L.E.

Vandenwijngaert, Sara

Vandersteen, Anthony

VanderWeele, David

Vandooren, Jennifer

Vanella, Luca

Vapaatalo, Heikki

Vaporidi, Katerina

Varadarajan, Sridhar

Varallyay, Eva

Varela-López, Alfonso

Vargiu, Attilio V.

Vashee, Sanjay

Vashist, Yogesh K.

Vasquez, Elisardo

Vassilopoulos, George

Vatta, Matteo

Vaughn, Byron

Vaz, Deisi Altmajer

Vecchione, Carmine

Vecoli, Cecilia

Vedel, Anne G.

Velasco Ortega, Eugenio

Veldhuizen, Edwin

Veleeparambil, Manoj

Velkov, Tony

Velliyagounder, Kabilan

Velotti, Francesca

Venditti, Alessandro

Venegas-Calerón, Mónica

Venkatesh, Jelli

Venkatesha, Shivaprasad

Venkitachalam, Srividya

Venter, Rietie

Venturi, Francesca

Venuti, Aldo

Verdoucq, Lionel 
Vergara, Daniele

Vergauwen, Lucia

Verhasselt, Bruno

Verhoeven, Adrie J.

Verrecchia, Franck

Verri, Tiziano

Verron, Elise

Verstuyf, Annemieke

Vetvicka, Vaclav

Vicente-Carrillo, Alejandro

Viejo-Borbolla, Abel

Viel, Tânia Araújo

Viennois, Emilie

Vierra, Craig

Viggiano, Davide

Vignard, Julien

Vik-Mo, Einar Osland

Vilanova, Manuel

Villanneau, Richard

Vinciguerra, Manlio

Vink, Robert

Vinnedge, Lisa Privette

Viruel, Maria Angeles

Visioli, Francesco

Vitetta, Luis

Vitorino, Rui

Vittori, Sauro

Vittorioso, Paola

Vizan, Pedro

Vladimirov, Vladimir I.

Vlase, Laurian

Vlashi, Erina

Vodnar, Dan Cristian

Voelkers, Mirko

Vogel, Ulla

Vogelaar, Christina Francisca

Volk, David

Volk, Martin

Volz, David C.

Von Brunn, Albrecht

Von Eggeling, Ferdinand

Von Knethen, Andreas

Vonk, Lucienne A.

Voss, Matthias

Vougas, Kostas

Vuckovic, Dajana

Vuppalanchi, Raj

Vyas, Maulik

Waalen, Jill

Wabiko, Hiroetsu

Wada, Jun

Wada, Nikolas I.
Wada, Ryuichi

Wade, Scott

Wadehra, Madhuri

Wadhwa, Sunil

Wagenmakers, A.J.M.

Waggoner, Stephen N.

Wagner, Anika

Wagner, Brent $\mathrm{T}$.

Wagner, Bridget K.

Wagner, David

Wähälä, Kristiina

Wahlestedt, Claes

Wahli, Walter

Wakabayashi, Ichiro

Wakamatsu, Kazumasa

Wakisaka, Naohiro

Waldau, Ben

Waldmeier, Felix

Walker, William B., III

Walker, Douglas G.

Walker, Heather

Walker, Jessica

Walker, M.M.

Wallace, Heather

Walsby, Charles J.

Walser, Tonya C.

Walsh, David

Walters, Dianne

Walther, Dirk

Wan, Dehui

Wan, Xuehua

Wandosell, Francisco

Wang, Aijun

Wang, Bo-Cheng

Wang, Ching-Chiung

Wang, Chin-Kun

Wang, Chrong-Reen

Wang, Chuanfeng

Wang, Dan

Wang, David

Wang, Feng-Sheng

Wang, Huanyou

Wang, Huei-Ju

Wang, Hui

Wang, Jeh-Jeng

Wang, Jian

Wang, Jian-Wen

Wang, Jianying

Wang, Joshua

Wang, Juan

Wang, Jun

Wang, Junbo
Wang, Junjie

Wang, Kai

Wang, Kevin Yueju

Wang, Le

Wang, Ling Zhi

Wang, Maxwell

Wang, Peng

Wang, Qi

Wang, Qiming

Wang, Qun

Wang, Raymond Y.

Wang, Robert Y.-L.

Wang, Rui

Wang, Ruoxiang

Wang, Shao-Chun

Wang, Sheng

Wang, Shibo

Wang, Tian-Li

Wang, Ting-Fang

Wang, Tzu-Wei

Wang, Wei-Lung

Wang, Xiaohong

Wang, Xin

Wang, Ying

Wang, Yumeng

Wang, Yun-Xing

Wang, Zhanwei

Wang, Zhijie

Wang, Zhixiang

Wang, Zhizhi

Wang, Zongwei

Wangchuk, Phurpa

Wanke, Dierk

Warby, Simon C.

Ward, Peter A.

Wary, Kishore K.

Watanabe, Masakatsu

Watanabe, Masatoshi

Watanabe, Masayuki

Watanabe, Mineo

Watanabe, Yuichiro

Watchko, Jon

Waterhouse, Miguel

Waters, Michael

Waters, Michael J.

Waters, Paddy

Watkins, Adam J.

Watkins, G. Leonard

Watt, Suzanne

Watzka, Matthias

Waugh, Mark G.

Waxman, David J. 
Waxman, Stephen G.

Weber, Daniel Gilbert

Weber, Franz E.

Wee, Joseph T.S.

Wegner, Marthe-Susanna

Wei, Andrew

Wei, Changyong

Wei, Gan

Wei, Jianjun

Wei, Sainan

Wei, Shau-Ming

Wei, Sheng

Wei, Yufeng

Weiergräber, Marco

Weigand, Annika

Weigand, Julia E.

Weiser, Douglas C.

Weisleder, Noah

Weljie, Aalim

Welsch, Ralf

Wen, C.M.

Wen, Xiaomin

Wendell, Stacy Gelhaus

Weng, Ching-Feng

Werling, Dirk

Werner, Inge

Werner, Thomas

Werstuck, Geoff H.

Wertz, Philip W.

Westenfelder, Christof

Westhoff, Andrew

Westin, Gunnar

Weström, Björn

Westwell, Andrew

Wetzel, Christian

Wheeler, Thomas

Whipple, C.A.

Whisstock, James C.

White, Peter

White, Thomas A.

Whitehurst, Angelique W.

Whysall, Kasia

Wiborg, Ove

Widgerow, Alan D.

Wie, Myung-Bok

Wieben, Oliver

Wiese, Stefan

Wigle, J.T.

Wijburg, Frits

Wilber, Andrew

Wilhelm, Stefan

Wilkins, Laetitia G.E.
Willerth, Stephanie M.

Willför, Stefan

Williams, Andrew R.

Williams, Bart O.

Williams, David

Williams, Jack D.

Williams, James

Williams, Nicole

Williams, Thomas L.

Williams, Tiffany

Williamson, Mike

Willison, Hugh

Willoughby, Darryn

Wilson, Anthony G.

Wilson, Brenda Anne

Wilson, David B.

Wilson, Douglas

Wilson, Heather

Wilson, Richard

Winger, Quinton

Winkler, David A.

Wirth, Michael

Wirths, Oliver

Wise, John

Witczak, Carol

Witeska, Małgorzata

Witorsch, Raphael Jay

Witt-Enderby, Paula

Witting, Michael

Wittung-Stafshede, Pernilla

Wolfe, Andrew

Wolff, A.S.B.

Wölfler, Albert

Wolosin, J. Mario

Wolschendorf, Frank

Woltering, Ernst

Wong, Boon-Seng

Wong, Chung F.

Wong, Connie H.Y.

Wong, George K.C.

Wong, Grace Lai-Hung

Wong, Ka-Chun

Wong, Kenneth K.Y.

Wong, Michale Ning

Wong, Nicholas C.

Wong, Ronald J.

Wong, Sunny $\mathrm{H}$.

Wong, Vincent

Wood, Christopher

Wood, Geoffrey A.

Wooddell, Christine I.

Woodruff, Prescott
Woods, Nicholas

Wooten, Joshua S.

Worrall, Margaret

Wower, Jacek

Wronska, Anetta

Wrzaczek, Michael

Wu, Chuanlong

$\mathrm{Wu}, \mathrm{Hao}$

Wu, Huaizhu

Wu, Jin-Yi

$\mathrm{Wu}$, Lianfeng

$\mathrm{Wu}$, Peter $\mathrm{K}$.

$\mathrm{Wu}$, Qian

$\mathrm{Wu}$, Sheng-Nan

Wu, Shih-Hsiung

$\mathrm{Wu}$, Tzong-Yuan

$\mathrm{Wu}$, Xiaoqing

Wullaert, Andy

Wurtman, Richard J.

Wydra, Kerstin

Wymann, Matthias

Xafenias, Nikolaos

Xavier, Nuno Manuel

Xia, Tian

Xian, Wa

Xiang, Dongxi

Xiang, Yu

Xiao, Junhua

Xiao, Li

Xiao, Zhousheng

Xie, Jianping

Xie, Jinghang

Xie, Maohua

Xie, Qi

Xie, Shaojun

Xie, Yang

Xin, Dongyue

Xin, Mei

Xing, Fei

Xirodimas, Dmitris

$\mathrm{Xu}$, Baoshan

Xu, Haineng

$\mathrm{Xu}$, Jian-Nong

Xu, Keli

Xu, Pengfei

Xu, Wei

$X u$, Xin

Xu, Yanyi

$\mathrm{Xu}$, Yiru

Xuan, Tran Dang

Xuan, Xiangchun

Xue, Xiang 
Yachida, Shinichi

Yadav, Pankaj

Yaegashi, Hajime

Yagi, Akira

Yagi, Fumio

Yakovlev, Igor A.

Yamada, Shuhei

Yamada, Sohsuke

Yamakawa-Kobayashi, K.

Yamaki, Kohji

Yamamoto, Kei

Yamamura, Soichiro

Yamanaka, Koji

Yamanishi, Kiyofumi

Yamasu, Kyo

Yamato, Masayuki

Yamauchi, Akira

Yamauchi, Takahiro

Yamawaki, Hideyuki

Yan, Bowen

Yan, Leping

Yan, Liang

Yan, Lin

Yandeau-Nelson, Marna D.

Yang, Chang-Hao

Yang, Ding-I

Yang, Dongli

Yang, Hung-Wei

Yang, Jenq-Lin

Yang, Jianhua

Yang, Mihi

Yang, Shengyong

Yang, Sze-Ming Mildred

Yang, Tao

Yang, Tsung-Lin

Yang, Wei-Hsiung

Yang, Xue

Yang, Yanan

Yang, Ye

Yang, Yongguang

Yang, Yu-Chiao

Yang, Yunze

Yano, Eizi

Yano, Shozo

Yao, Jian

Yao, Jiayi

Yao, Wei

Yao, Yanhua

Yao, Zhong-Ping

Yarotskyy, Viktor

Yassin, M.A.

Yasuchika, Kentaro
Yasuda, Kazuki

Yasuo, Shinobu

Yasutomi, Yasuhiro

Yazlovitskaya, Eugenia M.

Ye, Lin

Ye, Sang-Ho

Ye, Zhou

Yeh, Chau-Ting

Yeh, Chih-Ko

Yeh, Jan-Ying

Yeh, Jwu-Lai

Yeh, Shu-Lan

Yeh, Wei-Lan

Yeh, Yin-Ting

Yellen, Benjamin B.

Yen, Feng-Lin

Yen, Jeng-Hsien

Yin, Kingsley

Yin, Shi

Yin, Zhongchao

Yndestad, Arne

Yochum, Gregory S.

Yokoi, Kakeru

Yokoi, Tsuyoshi

Yokosuka, Akihito

Yokota, Shinso

Yokoyama, Tomoyuki

Yoneda, Toshiyuki

Yonekura, Masami

Yonekura, Shinichi

Yoo, Soonmoon

Yoo, Tag Keun

Yool, Andrea J.

Yoon, Do-Young

Yoon, Gyesoon

Yoon, Je-Hyun

Yorgason, Jordan

Yoshida, Hiroshi

Yoshida, Naoko

Yoshida, Takashi

Yoshihito, Yokoyama

Yoshimura, Akihiko

Yoshinaga, Kazuya

Yoshino, Jun

Yoshitomi, Hiroyuki

Yoshiyama, Hironori

You, Xiaona

You, Zongbing

Youn, Buhyun

Young, David

Yousaf, Muhammad Naveed

$\mathrm{Yu}$, Cheng-Chia
Yu, Cheng-Ping

$\mathrm{Yu}$, Chengzhong

Yu, Chia-Li

Yu, Ching-Ching

$\mathrm{Yu}$, Fengqun

Yu, Jaecheul

Yu, Richard

Yu, Yan Ping

Yu, Yinghua

Yu, Zhanyang

Yuan, Chun

Yuan, Shinsheng

Yuan, Xue

Yuan, Zhao

Yuan, Zhihong

Yuasa, S.

Yue, PatrickYingKit

Yue, Xiaojing

Yuk, Hyun-Gyun

Yumoto, Hiromichi

Yusuf, Nabiha

Zabel, Brian A.

Zaccai, Michele

Zachut, Maya

Zago, Miriam

Zakharian, Eleonora

Zambrano, Elena

Zamyatnin, Andrey

Zandi, Keivan

Zandi, Omid

Zanetti, Michela

Zang, Liqing

Zanin, Laura

Zaphiropoulos, Peter

Zaramella, Patrizia

Zarbin, Marco

Zaunders, John

Zavattaro, Elisa

Zavitsas, Andreas

Zawilska, Jolanta B.

Zehbe, Kerstin

Zehl, Martin

Zeller, Sebastian

Zemel, Michael

Zempleni, Janos

Zenclussen, Ana

Zeng, Huaqiang

Zerbini, Gianpaolo

Zeugolis, Dimitrios

Zgaga, Lina

Zhang, Bin

Zhang, Caiguo 
Zhang, Christie

Zhang, Deqiang

Zhang, Donglin

Zhang, Duo

Zhang, Jianping

Zhang, Jie

Zhang, Junzeng

Zhang, Li

Zhang, Mingliang

Zhang, Peng

Zhang, Qiuyang

Zhang, Ruiqin

Zhang, Tao

Zhang, Wei

Zhang, Wenheng

Zhang, Wenjun

Zhang, Xin

Zhang, Xuan

Zhang, Xuchen

Zhang, Yan-Bo

Zhang, Yanpeng

Zhang, Yixiang

Zhang, Yixuan
Zhang, Yuanyuan

Zhang, Zhengang

Zhao, Liang

Zhao, Meixia

Zhao, Ningning

Zhao, Qian

Zhao, Xiaoai

Zheng, Shilong

Zheng, Yue

Zheng, Zhi-Ming

Zhong, Tuhua

Zhou, Donghua H.

Zhou, Jia

Zhou, Qingxiang

Zhou, Wenchao

Zhou, Xiaoming

Zhou, Zhengping

Zhu, Fang

Zhu, Hailong

Zhu, Hai-Long

Zhu, Jianhua

Zhu, Liandong

Zhu, Yefei
Zhu, Yuwen

Zhu, Zhe

Ziman, Mel

Zimmer, Phillip

Zimring, James C.

Zingg, Jean-Marc

Zoja, Carlamaria

Zöller, Margot

Zong, Sheng Guo

Zorzano, Antonio

Zou, Shengli

Zou, Xueqing

Zucchi, Ileana

Zuluaga, Paola

Zünkler, Bernd-Joachim

Zuo, Li

Zupko, Istvan

Zusso, Morena

Zwarthoff, Ellen C.

Zweckstetter, Markus

Zytynska, S.E.

(C) 2017 by the authors; licensee MDPI, Basel, Switzerland. This article is an open access article distributed under the terms and conditions of the Creative Commons Attribution (CC-BY) license (http://creativecommons.org/licenses/by/4.0/). 